\title{
Optimal Maintenance Modeling for Systems with Multiple Non-Identical Units Using Extended DSSP Method
}

\author{
Xiaohong Zhang1,2, Jianchao Zeng1,3* \\ ${ }^{1}$ Division of Industrial and System Engineering, Taiyuan University of Science \& Technology, \\ Taiyuan, China \\ ${ }^{2}$ School of Economics \& Management, Taiyuan University of Science \& Technology, Taiyuan, China \\ ${ }^{3}$ School of Computer Science and Control Engineering, North University of China, Taiyuan, China \\ Email: zhangxiaohong1111@126.com, *zengjianchao@263.net
}

Received 9 April 2016; accepted 27 June 2016; published 30 June 2016

Copyright (C) 2016 by authors and Scientific Research Publishing Inc.

This work is licensed under the Creative Commons Attribution International License (CC BY).

http://creativecommons.org/licenses/by/4.0/

(c) (i) Open Access

\section{Abstract}

In the optimal maintenance modeling, all possible maintenance activities and their corresponding probabilities play a key role in modeling. For a system with multiple non-identical units, its maintenance requirements are very complicated, and it is time-consuming, even omission may occur when enumerating them with various combinations of units and even with different maintenance actions for them. Deterioration state space partition (DSSP) method is an efficient approach to analyze all possible maintenance requirements at each maintenance decision point and deduce their corresponding probabilities for maintenance modeling of multi-unit systems. In this paper, an extended DSSP method is developed for systems with multiple non-identical units considering opportunistic, preventive and corrective maintenance activities for each unit. In this method, different maintenance types are distinguished in each maintenance requirement. A new representation of the possible maintenance requirements and their corresponding probabilities is derived according to the partition results based on the joint probability density function of the maintained system deterioration state. Furthermore, focusing on a two-unit system with a non-periodical inspected condition-based opportunistic preventive-maintenance strategy; a long-term average cost model is established using the proposed method to determine its optimal maintenance parameters jointly, in which "hard failure" and non-negligible maintenance time are considered. Numerical experiments indicate that the extended DSSP method is valid for opportunistic maintenance modeling of multi-unit systems.

\footnotetext{
*Corresponding author.
}

How to cite this paper: Zhang, X.H. and Zeng, J.C. (2016) Optimal Maintenance Modeling for Systems with Multiple NonIdentical Units Using Extended DSSP Method. American Journal of Operations Research, 6, 275-295 


\section{Keywords}

\section{Extended Deterioration State Space Partition (DSSP), Condition-Based Opportunistic Preventive-Maintenance, Hard Failure, Non-Negligible Maintenance Times, Multi-Unit Systems}

\section{Introduction}

Preventive maintenance (PM) is a broad term that includes a set of activities to improve the overall reliability and availability of a system [1]. Many of today's technological systems, such as aircrafts, nuclear power plants, military installations and advanced industrial and medical equipments, are comprised of multiple non-identical critical units. It involves high level of complexity in their maintenance and operation, due to interactions between multiple units within a system. It is very important to design and implement appropriate and effective preventive maintenance for them. On one hand, these interactions must be taken into account in the maintenance decisions because of their strong influences. On the other hand, the interactions also offer the opportunity to group maintenance actions, which may save costs of system performance. Furthermore, improvements in analytical techniques and the availability of fast computers have allowed more complex systems to be investigated [2]. Over the past decades, there has been a growing interest in the modeling and optimization of maintenance of systems consisting of multiple units. As can be seen in the related review papers [2]-[6].

According to the literature, opportunistic maintenance is an effective maintenance strategy for multi-unit systems to collect units in a system that aims to reduce maintenance costs by grouping the maintenance activities of two or more units. Although the goal of opportunistic maintenance is to reduce maintenance costs, it may also impact plant availability. Therefore, the opportunistic maintenance policy was gradually studied in various multi-unit systems maintenance modeling based on different maintenance strategies, such as age-based maintenance (ABM) strategy [7], failure-rate-tolerance-based maintenance (FBM) strategy [8] and condition-based maintenance (CBM) strategy [9].

In the optimization maintenance model, all possible maintenance requirements and their corresponding probabilities play an essential role in modeling. For a multi-unit system using opportunistic maintenance strategy, maintenance requirements can be dynamic groups with different combination of the system units, even with different maintenance actions for these units. In order to formulate the decision problem of multi-unit systems with less mathematical difficulty, most of previous optimal preventive maintenance models for multi-unit systems were developed by means of some simple modeling method, such as Monte Carlo simulation techniques [10] [11]. And in these models, enumeration method is usually used to list all maintenance activities of the system. However, for a system with multiple units, simulation and enumeration method are more complicated and time-consuming, even omission may occur when enumerating its possible maintenance activities. Therefore, it needs to find a more efficient method to analyze the maintenance requirements and deduce their corresponding probabilities for these systems.

For a system composing $n$ units, its deterioration space is an $n$-dimensional continuous state space. With the increasing of the number of units, the combination of the system states and its calculation for modeling grow exponentially. To deal with curse of dimensionality, many previous researched decomposes a multi-unit system into mutually influential single-unit systems and each single-unit system is formulated separately. Then, it developed an approximation algorithm to obtain an acceptable maintenance policy for a multi-unit system, such as the studies by Wijnmalen and Hontelez [8], Jinqiu, H. and Z. Laibin [12] and Z. Zhang et al. [13]. However, if dependences between units are considered, the optimal maintenance decision on the whole system should depend on the deterioration state of the system rather than the deterioration state of individual units. That is to say, optimal maintenance of the individual unit cannot ensure the optimal maintenance of the whole system. Therefore, the decomposing method will injured much inaccuracy and cannot get an efficient maintenance policy since it can reduce the dimension of problem.

According to the above analysis, a general representation of the system deterioration should be presented for a general multi-unit system to characterize the deterioration of the whole system. Deterioration state space partition (DSSP) method is an efficient approach proposed in our previous studies for maintenance modeling of general multi-unit systems with identical [14] and non-identical units [15]. In these methods, all possible mainten- 
ance requirements at each maintenance decision point are analyzed and their corresponding probabilities are deduced according to the partition results of the joint deterioration state of units. After then an explicit representation of the stationary law of the whole system deterioration is derived and its numerical solution is developed considering the dependences between units. Instead of giving an objective-specific optimization model (such as reliability, availability and cost rate or multi-objective), these studies only provide a generalized modeling method for maintenance optimization of multi-unit systems. Also for simplification, the partition method in the recent study [15] only considered whether the unit should or not be maintained to model its deterioration state transition for a non-identical multi-unit system. However, for an objective-specific optimization model, it should not only distinguish which unit should be maintained, also which type of maintenance (opportunistic, preventive or corrective) should be performed on it due to their difference maintenance properties, such as cost and time. Therefore, a more detailed partition method should be studied to satisfy this modeling requirement.

In this paper, an extended DSSP method is presented for opportunistic maintenance modeling of general non-identical multi-unit systems by distinguishing the maintenance type of each unit. In the improved method, a more detailed partition is analyzed and new representation of the possible maintenance groups and their corresponding probabilities are derived based on it. Furthermore, in order to show the implementing process of the developed method, a non-periodical inspected condition-based optimization maintenance model is developed for a two-unit system considering hard failure and the non-negligible maintenance times. Using the proposed method, a stochastic model for the long-term average cost per unit time is developed based on semi-regenerative process theory to determine the optimal inspection interval and control limits of units jointly.

The remaining sections are organized as follows. Section 2 describes the extended DSSP method. In Section 3, using the proposed method, a cost model is proposed to assess and optimize the performance of the maintenance policy for two-unit system. Section 4 presents a numerical example, where the analysis results show the implementing process of the developed method. Finally, Section 5 concludes the paper.

\section{Extended DSSP Methods}

The opportunistic maintenance policy was gradually used in various multi-unit systems maintenance modeling based on different maintenance strategies. In the previous proposed opportunistic maintenance strategy studies, a zone of "opportunistic maintenance" such as the age range $\left(n_{i}, N\right)$ [7], the failure rate interval $(L-u, L)$ [8] and the deterioration level interval $\left[\varsigma^{(j)}, \xi_{k}^{(j)}\right)$ [9], is typically defined according to the control-limit policy to determine which units should be opportunistically maintained given that a maintenance action on other unit is performed. For multi-unit systems with non-identical units, DSSP method is present in paper [15] to model the opportunistic maintenance of them. In the method, various maintenance actions are corresponded to various deterioration state zones which are formed by splitting the deterioration state space of each unit with maintenance thresholds. For a system consisting of two or more units, the partition result of the system deterioration state is the crossover of the maintenance zone of every unit. More zones present more possible combinations of maintenance actions. In this pare, all possible maintenance groups of general multi-unit systems with a known number of non-identical units at each maintenance decision time and their corresponding probabilities are deduced using the presented approach. And further, a general representation of the stationary law of the system deterioration and its numerical solution is developed. Taking the common feature of the opportunistic maintenance policy into account, an extended DSSP method for opportunistic maintenance of multi-unit systems is proposed as below.

\subsection{Characteristics of System}

A multi-unit system with $n$ non-identical units is considered, in which each unit deteriorates gradually. The general assumptions for the system are as follows:

1) The deterioration state of unit $i(i=1,2, \cdots, n)$ at time $t$ can be described by a scalar random variable $x_{t}^{(i)}$ with initial state $x_{0}^{(i)}=0$, which means the unit $i$ is a new one. The unit $i$ is considered failed as soon as its deterioration state exceeds a critical level $D_{f}^{(i)}$.

2) Let $\mathbf{X}_{i}(t)$ be the continuous stochastic process describing the deterioration process of unit $i$. On an infinite discrete time grid $t_{k}(k \in \mathrm{N})$, the stochastic process can be described as $\left(\mathbf{X}_{k}^{(i)}\right)_{k \in \mathrm{N}}$. 
3) The increment of deterioration state of unit $i$ between two consecutive time units (i.e., between $t_{k-1}$ and $t_{k}$ ), $\Delta x_{k}^{(i)}=x_{k}^{(i)}-x_{k-1}^{(i)}$, is supposed to be nonnegative, stationary, and statistically independent, which follows the same distribution and the probability density function is $f_{i}(x)$. Consequently, the distribution of the increments during $n$ units of time is $f_{i}^{(n)}(x)$, where $f_{i}^{(n)}(x)$ is the $n$th convolution of $f_{i}(x)$.

4) The global system deterioration evolution is modeled by the $n$-dimensional continuous stochastic process $\mathbf{X}(t)=\left(\mathbf{X}_{1}(t), \cdots, \mathbf{X}_{n}(t)\right)$. The deterioration state of the system at time $t$ can be described by $n$-dimensional random variable $\left(x_{t}^{(1)}, \cdots, x_{t}^{(n)}\right)$ and it be simplified as $\left(x_{1}, \cdots, x_{n}\right)$ in the following.

The maintenance strategy is based on a control limit policy (CLP), which combines condition-based preventive, opportunistic and corrective maintenance. For each unit $i(i=1,2, \cdots, n)$, the thresholds $D_{p}^{(i)}$ and $D_{o}^{(i)}$ are defined for preventive maintenance and opportunistic maintenance respectively. It is generally assumed that $0 \leq D_{o}^{(i)} \leq D_{p}^{(i)} \leq D_{f}^{(i)}$. The detailed strategies are as follows:

1) Each unit is inspected non-periodically at discrete times $t_{k}(k \in \mathrm{N})$, where $k$ is the number of inspection and $\tau_{k}$ is the interval of the $k$ th inspection. After each inspection, the exact maintenance activities are determined according to the deterioration state at that time, which is denoted as $x_{i}$.

2) If $D_{p}^{(i)} \leq x_{i}<D_{f}^{(i)}$, the unit $i$ is maintained preventively.

3) If $x_{i} \geq D_{f}^{(i)}$, an corrective maintenance is performed on the unit $i$.

4) At each maintenance decision point, if a maintenance is performed on a unit $i$, for another unit $j(j \neq i)$, if its deterioration state satisfies $D_{o}^{(j)} \leq x_{j}<D_{p}^{(j)}$, it is maintained opportunistically together with unit $i$.

5) Otherwise, the units are left as they are.

After maintenance, the maintained unit can be restored to "as good as new" state.

\subsection{The Extended DSSP Method}

According to the defined strategy, Figure 1 illustrates the deterioration evolution of a single unit system. In this figure, the three maintenance thresholds of each unit split its deterioration state space into four zones: operating zone $(\mathrm{U})$, opportunistic maintenance zone $(\mathrm{O})$, preventive maintenance zone $(\mathrm{P})$ and corrective maintenance zone (C). Each zone presents one maintenance action. Each zone presents one maintenance action. Therefore, the partition result of the deterioration state space of a single-unit system can be simplified as Figure 2 with distinguishing different maintenance types.

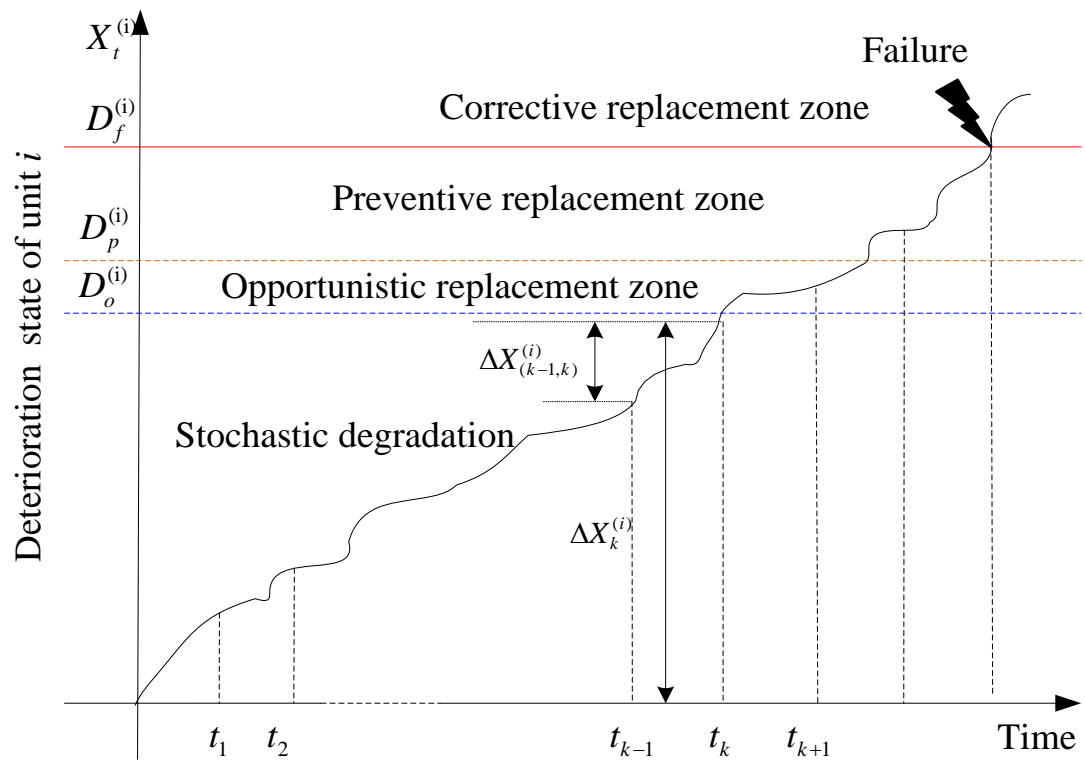

Figure 1. Deterioration model of the $i^{\text {th }}$ unit. 




Deterioration stateof unit1

Figure 2. Deterioration state space partition of single-unit systems.

For a system consisting of two or more units, the partition result of the system deterioration state is the crossover of the maintenance zone of every unit. Each zone presents a possible combination of maintenance actions of all units. The deterioration state space partition with all possible maintenance requirements in two-unit systems and three-unit systems are depicted in Figure 3 and Figure 4. In Figure 4, the partition result is divided into four subparts in accordance with four different maintenance requirements of the third unit: no maintenance (Figure 4(a)), opportunistic maintenance (Figure 4(b)), preventive maintenance (Figure 4(c)) and corrective maintenance (Figure 4(d)). In these figures, each deterioration state space region is named by a maintenance activity list which represents the maintenance requirement for each unit respectively.

For a multi-unit systems with $n$ non-identical units, its possible maintenance requirement can be expressed as a maintenance activity list $A_{1} A_{2} \cdots A_{n}$, where $A_{i}$ denotes the maintenance activity for unit $i$ and it have four possible scenarios: $\mathrm{U}, \mathrm{O}, \mathrm{P}$ and $\mathrm{C}$. Therefore, there are $4^{n}$ possible maintenance requirements for a $n$-unit system corresponding with $4^{n}$ deterioration state space regions. It's easy to find that the possible maintenance requirement groups of $n$-unit system can be derived from the possible maintenance requirements of $n-1$ units system by embedding its partition result to four possible scenarios: $\mathrm{U}, \mathrm{O}, \mathrm{P}$ and $\mathrm{C}$ of unit $n$. These possible maintenance activity lists are $A_{1} A_{2} \cdots A_{n-1} U, A_{1} A_{2} \cdots A_{n-1} O, A_{1} A_{2} \cdots A_{n-1} P$ and $A_{1} A_{2} \cdots A_{n-1} C$.

\subsection{Maintenance Probability}

Suppose $\pi_{n}\left(x_{1}, \cdots, x_{n}\right)$ be the joint probability density function of the system deterioration state $\left(x_{1}, \cdots, x_{n}\right)$ for a maintained multi-unit system with $n$ non-identical units. Let $P_{A_{1} A_{2} \cdots A_{n}}^{n}$ denote the probability of the maintenance requirement $A_{1} A_{2} \cdots A_{n}$. For a single-unit system, according to the partition in Figure 2, its probabilities of all possible maintenance requirements can be deduced by the integrating the probability density function of the deterioration state, $\pi_{1}\left(x_{1}\right)$, in its corresponding deterioration region, and the expressions can be expressed as Equations (1)-(4).

$$
\begin{aligned}
& P_{\mathrm{U}}^{1}\left(x_{1}\right)=\int_{0}^{D_{0}^{(1)}} \pi_{1}\left(x_{1}\right) \mathrm{d} x_{1} \\
& P_{\mathrm{O}}^{1}\left(x_{1}\right)=\int_{D_{0}^{(1)}}^{D_{p}^{(1)}} \pi_{1}\left(x_{1}\right) \mathrm{d} x_{1} \\
& P_{\mathrm{P}}^{1}\left(x_{1}\right)=\int_{D_{p}^{(1)}}^{D_{f}^{(1)}} \pi_{1}\left(x_{1}\right) \mathrm{d} x_{1} \\
& P_{\mathrm{C}}^{1}\left(x_{1}\right)=\int_{D_{f}^{(1)}}^{\infty} \pi_{1}\left(x_{1}\right) \mathrm{d} x_{1}
\end{aligned}
$$

The expressions of the probabilities of all possible maintenance requirements in two-unit systems also can be 


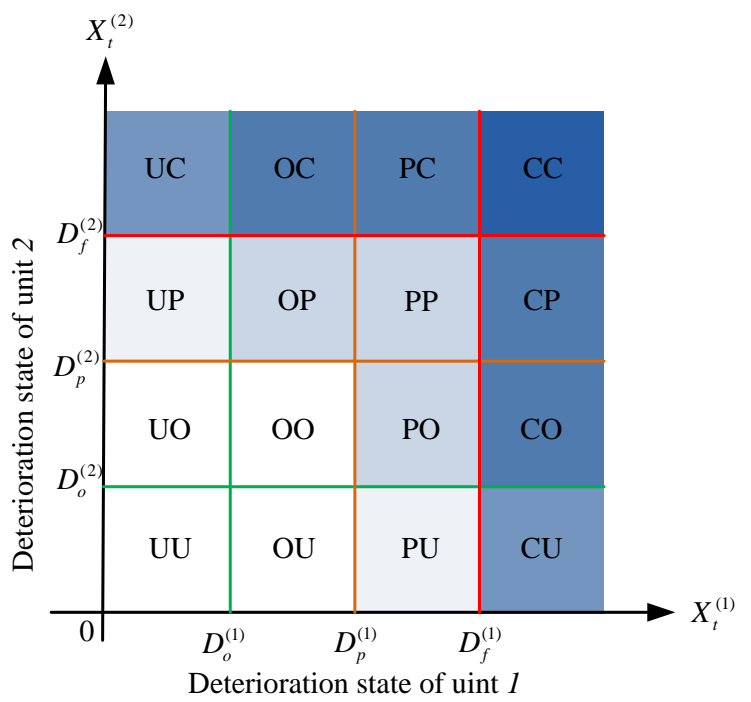

Figure 3. Deterioration state space partition of two-unit systems.



Deterioration state of unit 1

(a)

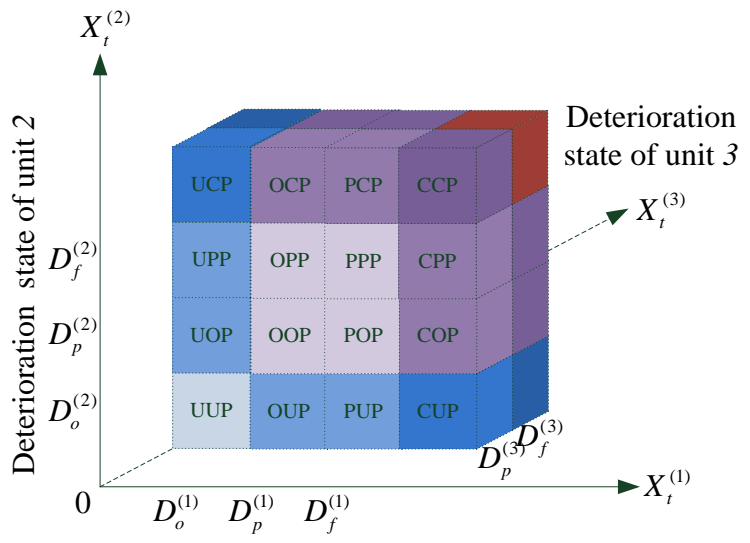

Deterioration state of unit 1

(c)

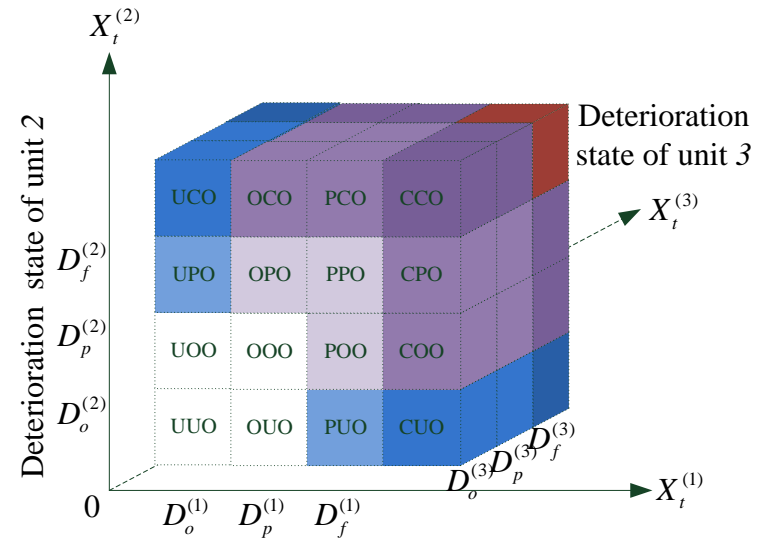

Deterioration state of unit 1

(b)

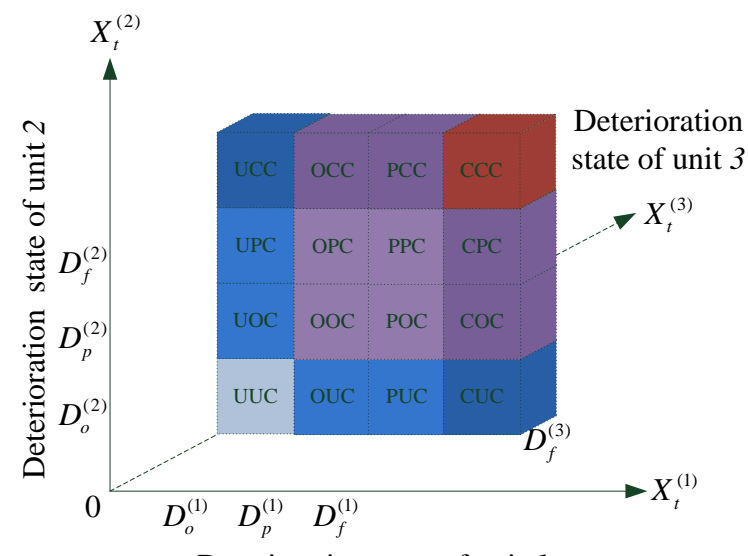

Deterioration state of unit 1

(d)

Figure 4. Deterioration state space partition of three-unit systems. (a) The third unit needs no maintenance; (b) The third unit needs opportunistic maintenance; (c) The third unit needs preventive maintenance; (d) The third unit needs corrective maintenance. 
presented by integrating the joint probability density function of the system deterioration state, $\pi_{2}\left(x_{1}, x_{2}\right)$, in its corresponding deterioration state space region according to the partition in Figure 3. All the probabilities of maintenance activities for a two-unit system are as shown in Equations (5)-(20).

$$
\begin{aligned}
& P_{\mathrm{UU}}^{2}\left(x_{1}, x_{2}\right)=\int_{0}^{D_{0}^{(2)}} \int_{0}^{D_{0}^{(1)}} \pi_{2}\left(x_{1}, x_{2}\right) \mathrm{d} x_{1} \mathrm{~d} x_{2} \\
& P_{\mathrm{UO}}^{2}\left(x_{1}, x_{2}\right)=\int_{D_{0}^{(2)}}^{D_{p}^{(2)}} \int_{0}^{D_{0}^{(1)}} \pi_{2}\left(x_{1}, x_{2}\right) \mathrm{d} x_{1} \mathrm{~d} x_{2} \\
& P_{\mathrm{UP}}^{2}\left(x_{1}, x_{2}\right)=\int_{D_{p}^{(2)}}^{D_{f}^{(2)}} \int_{0}^{D_{o}^{(1)}} \pi_{2}\left(x_{1}, x_{2}\right) \mathrm{d} x_{1} \mathrm{~d} x_{2} \\
& P_{\mathrm{UC}}^{2}\left(x_{1}, x_{2}\right)=\int_{D_{f}^{(2)}}^{\infty} \int_{0}^{D_{o}^{(1)}} \pi_{2}\left(x_{1}, x_{2}\right) \mathrm{d} x_{1} \mathrm{~d} x_{2} \\
& P_{\mathrm{OU}}^{2}\left(x_{1}, x_{2}\right)=\int_{0}^{D_{0}^{(2)}} \int_{D_{0}^{(1)}}^{D_{p}^{(1)}} \pi_{2}\left(x_{1}, x_{2}\right) \mathrm{d} x_{1} \mathrm{~d} x_{2} \\
& P_{\mathrm{OO}}^{2}\left(x_{1}, x_{2}\right)=\int_{D_{0}^{(2)}}^{D_{P}^{(2)}} \int_{D_{0}^{(1)}}^{D_{P}^{(1)}} \pi_{2}\left(x_{1}, x_{2}\right) \mathrm{d} x_{1} \mathrm{~d} x_{2} \\
& P_{\mathrm{OP}}^{2}\left(x_{1}, x_{2}\right)=\int_{D_{P}^{(2)}}^{D_{f}^{(2)}} \int_{D_{0}^{(1)}}^{D_{P}^{(1)}} \pi_{2}\left(x_{1}, x_{2}\right) \mathrm{d} x_{1} \mathrm{~d} x_{2} \\
& P_{\mathrm{OC}}^{2}\left(x_{1}, x_{2}\right)=\int_{D_{f}^{(2)}}^{\infty} \int_{D_{0}^{(1)}}^{D_{P}^{(1)}} \pi_{2}\left(x_{1}, x_{2}\right) \mathrm{d} x_{1} \mathrm{~d} x_{2} \\
& P_{\mathrm{PU}}^{2}\left(x_{1}, x_{2}\right)=\int_{0}^{D_{0}^{(2)}} \int_{D_{P}^{(1)}}^{D_{f}^{(1)}} \pi_{2}\left(x_{1}, x_{2}\right) \mathrm{d} x_{1} \mathrm{~d} x_{2} \\
& P_{\mathrm{PO}}^{2}\left(x_{1}, x_{2}\right)=\int_{D_{0}^{(2)}}^{D_{p}^{(2)}} \int_{D_{p}^{(1)}}^{D_{f}^{(1)}} \pi_{2}\left(x_{1}, x_{2}\right) \mathrm{d} x_{1} \mathrm{~d} x_{2} \\
& P_{\mathrm{PP}}^{2}\left(x_{1}, x_{2}\right)=\int_{D_{P}^{(2)}}^{D_{f}^{(2)}} \int_{D_{P}^{(1)}}^{D_{f}^{(1)}} \pi_{2}\left(x_{1}, x_{2}\right) \mathrm{d} x_{1} \mathrm{~d} x_{2} \\
& P_{\mathrm{PC}}^{2}\left(x_{1}, x_{2}\right)=\int_{D_{f}^{(2)}}^{\infty} \int_{D_{p}^{(1)}}^{D_{f}^{(1)}} \pi_{2}\left(x_{1}, x_{2}\right) \mathrm{d} x_{1} \mathrm{~d} x_{2} \\
& P_{\mathrm{CU}}^{2}\left(x_{1}, x_{2}\right)=\int_{0}^{D_{o}^{(2)}} \int_{D_{f}^{(1)}}^{\infty} \pi_{2}\left(x_{1}, x_{2}\right) \mathrm{d} x_{1} \mathrm{~d} x_{2} \\
& P_{\mathrm{CO}}^{2}\left(x_{1}, x_{2}\right)=\int_{D_{0}^{(2)}}^{D_{p}^{(2)}} \int_{D_{f}^{(1)}}^{\infty} \pi_{2}\left(x_{1}, x_{2}\right) \mathrm{d} x_{1} \mathrm{~d} x_{2} \\
& P_{\mathrm{CP}}^{2}\left(x_{1}, x_{2}\right)=\int_{D_{P}^{(2)}}^{D_{f}^{(2)}} \int_{D_{f}^{(1)}}^{\infty} \pi_{2}\left(x_{1}, x_{2}\right) \mathrm{d} x_{1} \mathrm{~d} x_{2} \\
& P_{\mathrm{OC}}^{2}\left(x_{1}, x_{2}\right)=\int_{D_{f}^{(2)}}^{\infty} \int_{D_{f}^{(1)}}^{\infty} \pi_{2}\left(x_{1}, x_{2}\right) \mathrm{d} x_{1} \mathrm{~d} x_{2}
\end{aligned}
$$

It is not difficult to induce that, for a multi-unit system with $n$ non-identical units, the partitions of the deterioration space of the $n$ units system can be constructed by embedding the partitions of the $n-1$ units system into the maintenance zones of the new unit. It can be known from Figure 2, there are four maintenance zones for the $n$th unit, operating zone $(\mathrm{U})$, opportunistic maintenance zone $(\mathrm{O})$, preventive maintenance zone $(\mathrm{P})$ and corrective maintenance zone $(\mathrm{C})$, and their coverage regions are $\left[0, D_{o}^{(n)}\right),\left[D_{o}^{(n)}, D_{p}^{(n)}\right),\left[D_{p}^{(n)}, D_{f}^{(n)}\right)$ and $\left[D_{f}^{(n)}, \infty\right)$ respectively. Therefore, the probability of maintenance requirement $A_{1} A_{2} \cdots A_{n}$ can be formed by the probability of maintenance activity list $A_{1} A_{2} \cdots A_{n-1}$ with four possible maintenance requirements of unit $n$. 
Their probabilities can be expressed as Equations (21)-(24).

$$
\begin{gathered}
P_{A_{1} A_{2} \cdots A_{n-1} \mathrm{U}}^{n}\left(x_{1}, x_{2}, \cdots, x_{n-1}, x_{n}\right)=\int_{0}^{D_{0}^{(n)}} \int_{Q_{Q_{n-1}}^{n-1}}^{n-1} \pi_{n}\left(x_{1}, x_{2}, \cdots, x_{n-1}, x_{n}\right) \mathrm{d} x_{1} \cdots \mathrm{d} x_{n-1} \mathrm{~d} x_{n} \\
P_{A_{1} A_{2} \cdots A_{n-1} \mathrm{O}}^{n}\left(x_{1}, x_{2}, \cdots, x_{n-1}, x_{n}\right)=\int_{D_{0}^{(n)}}^{D_{P}^{(n)}} \overbrace{\int_{Q_{n-1}}^{n-1} \int}^{\cdots-1} \pi_{n}\left(x_{1}, x_{2}, \cdots, x_{n-1}, x_{n}\right) \mathrm{d} x_{1} \cdots \mathrm{d} x_{n-1} \mathrm{~d} x_{n} \\
P_{A_{1} A_{2} \cdots A_{n-1} \mathrm{P}}^{n}\left(x_{1}, x_{2}, \cdots, x_{n-1}, x_{n}\right)=\int_{D_{p}^{(n)}}^{D_{f}^{(n)}} \overbrace{\int_{Q_{n-1}}^{n-1} \int}^{\cdots \int} \pi_{n}\left(x_{1}, x_{2}, \cdots, x_{n-1}, x_{n}\right) \mathrm{d} x_{1} \cdots \mathrm{d} x_{n-1} \mathrm{~d} x_{n} \\
P_{A_{1} A_{2} \cdots A_{n-1} \mathrm{C}}^{n}\left(x_{1}, x_{2}, \cdots, x_{n-1}, x_{n}\right)=\int_{D_{f}^{(n)}}^{\infty} \overbrace{\int \cdots \int}^{n-1} \pi_{n}\left(x_{1}, x_{2}, \cdots, x_{n-1}, x_{n}\right) \mathrm{d} x_{1} \cdots \mathrm{d} x_{n-1} \mathrm{~d} x_{n}
\end{gathered}
$$

where $Q_{n-1}$ denotes the deterioration state space region for maintenance activity list $A_{1} A_{2} \cdots A_{n-1}$.

A special case is that when there is no unit in the system need to be maintained, all the opportunistic maintenance requirements will be lay aside for no opportunity is offered. That is, in all regions crossover by operating zone $(\mathrm{U})$ and opportunistic maintenance zone $(\mathrm{O})$ of all units, there is no unit need maintenance. Let $M^{n}(0)$ denote the random event that there is no maintenance requirement in a multi-unit system with $n$ non-identical units, its probabilities can be expressed as:

$$
P_{M^{n}(0)}^{n}\left(x_{1}, x_{2}, \cdots, x_{n-1}, x_{n}\right)=\int_{0}^{D_{p}^{(n)}} \int_{0}^{D_{p}^{(n-1)}} \cdots \int_{0}^{D_{p}^{(1)}} \pi_{n}\left(x_{1}, x_{2}, \cdots, x_{n-1}, x_{n}\right) \mathrm{d} x_{1} \cdots \mathrm{d} x_{n-1} \mathrm{~d} x_{n}
$$

In a multi-unit system with $n$ non-identical units, the number of the regions crossover by operating zone (U) and opportunistic maintenance zone $(\mathrm{O})$ of all units is $2^{n}$, therefore, the really number of the possible maintenance requirement combinations is $4^{n}-2^{n}+1$

Equation (1) to Equation (25) indicate that the evaluation of such criterion requires determination of the joint probability density function of the deterioration evolution of the maintained system $\pi_{n}\left(x_{1}, \cdots, x_{n}\right)(n=1,2, \cdots)$. In our previous study [15], the general expression, numeric solution, experimental verification and analysis of $\pi_{n}\left(x_{1}, \cdots, x_{n}\right)(n=1,2, \cdots)$ have been introduced in detail. It will not be repeated in this paper.

\section{Optimal Maintenance Modeling for Two-Unit Systems}

In order to show the implementing process of the extended DSSP method, the optimization model of the maintenance for two-unit systems is established using the proposed method in this section.

\subsection{System Characteristics}

A two-unit system is considered in which each unit follows the general assumptions presented in Section 2.1. In addition, a consideration of hard failure is added. That is, the failure of each unit can be found immediately without inspections as it results in a system shutdown, which is referred as a hard failure [16]. In more detail, if the deterioration state of the unit $i$ satisfies $x_{i} \geq D_{f}^{(i)}$ at the time of inspection, an immediate corrective maintenance is performed on the failed unit [17]. If its deterioration state $x_{i}$ exceeds the critical level $D_{f}^{(i)}$ before a planning inspection, the system will down by itself, an immediate corrective maintenance is performed on the failed unit and the inspection plan is rearranged according to the deterioration state after maintained.

The maintenance strategy for this system is also based on the control limit policy defined in Section 2.2. Furthermore, non-periodical inspection intervals and non-negligible maintenance times are considered. More additional detailed assumptions are as follows:

1) Let $C_{i n s}$ be the specific unit cost for an inspection of the whole system. It consists of two parts, $C_{i n s}^{(1)}$ for unit 1 and $C_{\text {ins }}^{(2)}$ for unit 2. The inspection of a component is assumed as perfect and non-destructive, and the inspection time is considered negligible.

2) For unit $i(i=1,2)$, the maintenance cost incurred by corrective maintenance and preventive maintenance are constant values $C_{c}^{(i)}$ and $C_{p}^{(i)}$, and the maintenance time incurred by corrective maintenance and pre- 
ventive maintenance are $t_{c}^{(i)}$ and $t_{p}^{(i)}$ respectively. It can be generally considered that $C_{p}^{(i)} \ll C_{c}^{(i)}$ and $t_{p}^{(i)} \ll t_{c}^{(i)}$. When preventive maintenance or corrective maintenance is performed, the system is shutdown. The time elapsed by the system in shutdown state incurs a cost at a cost rate $C_{d}$.

3) It is assumed that the opportunistic maintenance of each unit incurs same cost and time as its preventive maintenance.

4) After each maintenance, a new inspection interval is rearranged.

Either a scheduled maintenance with periodic inspection and/or an unscheduled corrective maintenance for hard failure is considered as a system intervention. In addition to the specific unit cost as previously defined, each intervention performance on the system entails a set-up cost $C_{s}$, which is assumed to be independent of the nature of operation and incurred only once when several maintenance operations are performed at the same time.

\subsection{Evaluation of the Performance Criteria of the Proposed Policy}

According to the defined strategy, an example deterioration and maintenance evolution of a two-unit system is illustrated in Figure 5.

In this figure, $T_{k}(k \in \mathrm{N})$ are the scheduled inspection points. At the inspection time $T_{1}$ and $T_{3}$, no maintenance is required because there are no maintenance opportunities even though the deterioration states of the units belong to their opportunistic maintenance zones, the next inspection date for the system is scheduled according to the deterioration state at that time respectively. At the inspection time $T_{2}$, a preventive maintenance

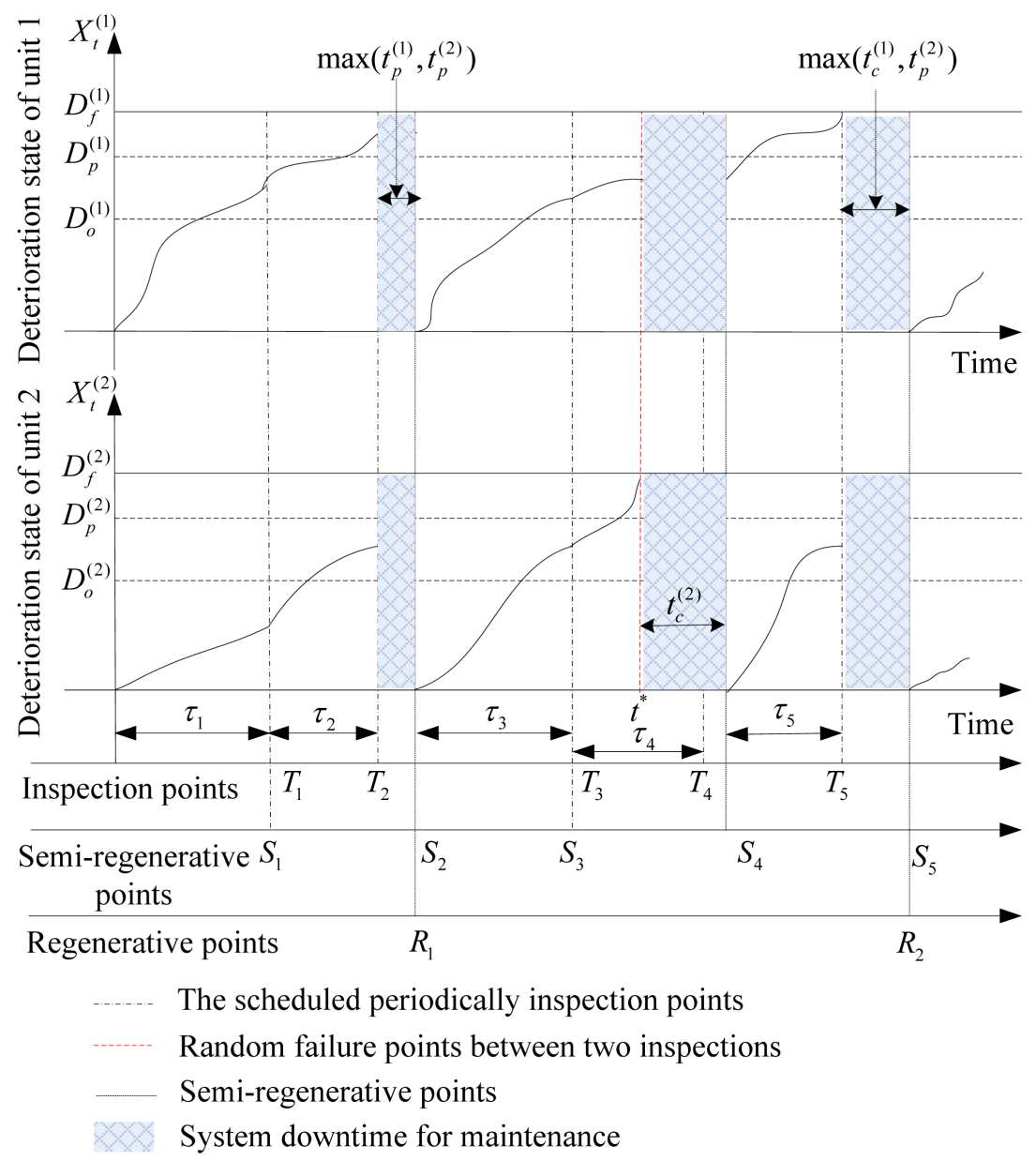

Figure 5. Example of deterioration and maintenance evolution of a two-unit system. 
is required for the unit 1 . While the deterioration state of unit 2 belongs to its opportunistic maintenance zone, it is opportunistically maintained together. After a certain maintenance interval, the system is restored to a new state and a new inspection cycle begins. At time $t^{*}$ between the inspection times $T_{3}$ and $T_{4}$, the deterioration state of unit 2 exceeds its critical level, and immediately a corrective maintenance is incurred. At that time, the system is shut down for maintenance. After maintenance, the system is also restored to "as good as new" state and an inspection is scheduled. At the inspection time $T_{5}$, a preventive maintenance is required for the unit 1 . The deterioration state of unit 2 is greater than its opportunistic maintenance threshold and opportunistic maintenance is required. It can be seen from Figure 5, at times $R_{1}$ and $R_{2}$, all of the units in the system are maintained and the system is restored to "as good as new" state. Thus, these points are denoted as regenerative points. However, at times $S_{2}, S_{4}$ and $S_{5}$, parts of system units are maintained and restored to their new states. Together with inspection points $S_{1}$ and $S_{3}$, all these points are denoted as semi-regenerative points.

It's easy to find that the length of a semi-regeneration cycle is varying stochastically. Let the stochastic variable $S$ be the length of a semi-regeneration cycle. It contains two time stochastic periods: 1) Uptime interval between the last starting up time and the system failure time or the next periodical inspection time, denoted as $S^{\prime}$, which varies stochastically with the stochastic failure and dynamic inspection interval. 2) Downtime for maintenance, denoted as $D$, which varies stochastically with the maintenance activities for stochastically deterioration.

A cost model is proposed to assess and optimize the performance of the proposed policy. According to renewal theory, the average cost of an infinite time is defined a,

$$
C R(t)=\lim _{t \rightarrow \infty} \frac{C(t)}{t} \approx \frac{\text { expected cost on one semi-renewal cycle }}{\text { expected length of a semi-renewal cycle }}=\frac{E(C(S))}{E(S)}
$$

where $E(S)$ is the expectation of the time length of a semi-regeneration cycle. The term $E(C(S))$ denotes the expectation of the total cost of the semi-regeneration cycle.

In accordance with the characteristics of the system deterioration process and the maintenance strategies described above, the choices of the inspection interval and the opportunistic and preventive maintenance thresholds for each unit influences the performance of the proposed policy. If the inspection interval is too large, the probability of failure increases between two inspections, which results in increased maintenance costs. In contrary, it is expensive to inspect the system too frequently. Similarly, the low opportunistic and preventive maintenance thresholds result in frequent preventive maintenance and the remaining life of the deteriorated but still working unit cannot be fully exploited. Otherwise, the failure probability and the maintenance costs increase between two inspections. To minimize the total maintenance cost of the whole system, the optimization of the proposed policy can be defined as a constrained optimization problem, if the average cost can be represented as a function of these decision variables as following:

$$
\begin{array}{ll}
\min & C R(t)=\min \Psi\left(\tau_{k}, D_{p}^{(1)}, D_{p}^{(2)}, D_{o}^{(1)}, D_{o}^{(2)}\right) \\
\text { s.t. } & \tau_{k}=m\left(x_{1}^{(k-1)}, x_{2}^{(k-1)}\right) \\
& D_{o}^{(1)} \leq D_{p}^{(1)} \leq D_{f}^{(1)} \\
& D_{o}^{(2)} \leq D_{p}^{(2)} \leq D_{f}^{(2)}
\end{array}
$$

The unit $i(i=1,2)$ is considered failed as soon as its deterioration state exceeds its critical level $D_{f}^{(i)}$, and the system is shutdown for the unit failure. Due to the characteristic of hard failure, the exact time of failure of unit $i$ is unknown. Therefore, the $k$ th stopping of the operation system can be two scenarios: 1) Pre-scheduled inspection, in which case the length of the $k$ th uptime duration is $S_{k}^{\prime}=\tau_{k}$. (2) System failure between two inspections, in which case the length of the $k$ th uptime duration is $S_{k}^{\prime}=\tau_{f}^{k}\left(0<\tau_{f}^{k}<\tau_{k}\right)$. It should be explain that if a failure occurs at time $\tau_{f}^{k}$ with $\tau_{f}^{k} \in\left(0, \tau_{k}\right]$. In order to facilitate the analysis, an infinite discrete time grid is adopted here, it is assumed that the exact time of failure of unit $i$ can be set as any one integer value between $\left(0, \tau_{k}\right]$. That is to say, it is assumed that that the exact failure time is at the integer discrete time if it occurs in a time unit. Let $H_{i}\left(\tau_{f}^{k} \mid y_{i}^{\prime} ; \tau_{k}\right)$ define the probability that the $k$ th failure time of component $i$ is $\tau_{f}^{k}$ time units 
after the last semi-regeneration point given that the revealed deterioration state equal $y_{i}^{\prime}$ and the next inspection was scheduled $\tau_{k}$ time units later (Castanier, Grall, \& Bérenguer, 2005). This probability can be expressed as,

$$
H_{i}\left(\tau_{f}^{k} \mid y_{i}^{\prime} ; \tau_{k}\right)= \begin{cases}0 & \text { if } \tau_{f}^{k}>\tau_{k} \\ f_{i}^{\tau_{f}^{k}}\left(D_{f}^{(i)}-y_{i}^{\prime}\right) & \text { if } \tau_{f}^{k} \leq \tau_{k}\end{cases}
$$

The expected uptime duration in a semi-regeneration cycle is expressed as:

$$
E\left(S^{\prime}\right)=\sum_{\tau_{f}^{k}=1}^{\tau_{k}-1} \tau_{f}^{k} P\left(S^{\prime}=\tau_{f}^{k}\right)+\tau_{k} P\left(S^{\prime}=\tau_{k}\right)
$$

Using $t_{f}$ as the exact failure time of the system, Equation (33) can be obtained.

$$
\begin{aligned}
P\left(S^{\prime}=\tau_{f}^{k}\right) & =P\left(\tau_{f}^{k}-1<t_{f} \leq \tau_{f}^{k}\right) \\
& =P\left(t_{f} \leq \tau_{f}^{k}\right) P\left(t_{f}>\tau_{f}^{k}-1\right) \\
& =P\left(t_{f} \leq \tau_{f}^{k}\right)\left[1-P\left(t_{f} \leq \tau_{f}^{k}-1\right)\right]
\end{aligned}
$$

The failure of the system can be triggered by failure of unit 1 and/or unit 2, therefore,

$$
P\left(t_{f}=r\right)=\sum_{i=1}^{2} P\left(t_{f}^{i}=r\right)-P\left(t_{f}^{1}=r, t_{f}^{2}=r\right)
$$

In Equation (31), $P\left(t_{f}^{i}=r\right)(i=1,2)$ consider the scenario that each unit in failure state separately, $P\left(t_{f}^{1}=r, t_{f}^{2}=r\right)$ takes into account the scenario that both units are simultaneously in failure state. These probabilities can be evaluated by analysis of all the scenarios leading to failure. The failure of unit $i$ may occur under two conditions. If unit $i$ was maintained at its previous semi-regenerative point, then $y_{i}^{\prime}=0$. If unit $i$ was not maintained at its previous semi-regenerative point, then $y_{i}^{\prime}=y_{i}<D_{p}^{(i)}$ and $y_{j}<D_{p}^{(i)}(i \neq j)$ or $y_{i}^{\prime}=y_{i}<D_{o}^{(i)}$ and $y_{j} \geq D_{p}^{(i)}(i \neq j)$. Therefore, the probability of the unit $i$ being in failure state is expressed as Equation (32), and the probability of both units being simultaneously in failure state is expressed as Equation (33).

$$
\begin{aligned}
P\left(t_{f}^{i}=r\right)=\left[\int_{D_{o}^{(2)}}^{\infty} \int_{D_{o}^{(1)}}^{\infty} \pi_{2}\left(y_{1}, y_{2}\right) \mathrm{d} y_{1} \mathrm{~d} y_{2}-\int_{D_{o}^{(2)}}^{D_{p}^{(2)}} \int_{D_{o}^{(1)}}^{D_{p}^{(1)}} \pi_{2}\left(y_{1}, y_{2}\right) \mathrm{d} y_{1} \mathrm{~d} y_{2}\right] H_{i}\left(r \mid 0, \tau_{k}\right) \\
+\left[\int_{0}^{D_{0}^{(j)}} \int_{D_{p}^{(i)}}^{\infty} \pi_{2}\left(y_{1}, y_{2}\right) \mathrm{d} y_{i} \mathrm{~d} y_{j}\right] H_{i}\left(r \mid 0, \tau_{k}\right)+\int_{0}^{D_{o}^{(i)}} \int_{D_{p}^{(j)}}^{\infty} \pi_{2}\left(y_{1}, y_{2}\right) H_{i}\left(r \mid y_{i}, \tau_{k}\right) \mathrm{d} y_{j} \mathrm{~d} y_{i} \\
+\int_{0}^{D_{p}^{(i)}} \int_{0}^{D_{p}^{(j)}} \pi_{2}\left(y_{1}, y_{2}\right) H_{i}\left(r \mid y_{i}, \tau_{k}\right) \mathrm{d} y_{j} \mathrm{~d} y_{i} \\
P\left(t_{f}^{1}=r, t_{f}^{2}=r\right)=\left[\int_{D_{0}^{(2)}}^{\infty} \int_{D_{0}^{(1)}}^{\infty} \pi_{2}\left(y_{1}, y_{2}\right) \mathrm{d} y_{1} \mathrm{~d} y_{2}-\int_{D_{o}^{(2)}}^{D_{p}^{(2)}} \int_{D_{o}^{(1)}}^{D_{p}^{(1)}} \pi_{2}\left(y_{1}, y_{2}\right) \mathrm{d} y_{1} \mathrm{~d} y_{2}\right] H_{1}\left(r \mid 0, \tau_{k}\right) H_{2}\left(r \mid 0, \tau_{k}\right) \\
+\left[\int_{0}^{D_{0}^{(2)}} \int_{D_{p}^{(1)}}^{\infty} \pi_{2}\left(y_{1}, y_{2}\right) \mathrm{d} y_{1} H_{2}\left(r \mid y_{2}, \tau_{k}\right) \mathrm{d} y_{2}\right] H_{1}\left(r \mid 0, \tau_{k}\right) \\
+\left[\int_{0}^{D_{0}^{(1)}} \int_{D_{p}^{(2)}}^{\infty} \pi_{2}\left(y_{1}, y_{2}\right) \mathrm{d} y_{2} H_{1}\left(r \mid y_{1}, \tau_{k}\right) \mathrm{d} y_{1}\right] H_{2}\left(r \mid 0, \tau_{k}\right) \\
+\int_{0}^{D_{p}^{(1)}} \int_{0}^{D_{p}^{(2)}} \pi_{2}\left(y_{1}, y_{2}\right) H_{1}\left(r \mid y_{1}, \tau_{k}\right) H_{2}\left(r \mid y_{2}, \tau_{k}\right) \mathrm{d} y_{2} \mathrm{~d} y_{1}
\end{aligned}
$$

If the system has not failed between two inspections, the operation of the system can be stopped by prescheduled inspection. Therefore, the probability of the length of the uptime duration is $S^{\prime}=\tau_{k}$ can be expressed as: 


$$
P\left(S^{\prime}=\tau_{k}\right)=1-\sum_{\tau_{f}^{k}=1}^{\tau_{k}-1} P\left(S_{k}^{\prime}=\tau_{f}^{k}\right)
$$

If the operation of the system is stopped by pre-scheduled inspection, all units in the system are inspected and the maintenance activities are arranged according to the revealed states. Different maintenance groups incur different probabilities and costs. According to partitions in Figure 3, the revealed system state can falls in 16 regions. Different cost will incurred for different regions with different probability which can be calculated according to to partitions in Figure 3, the revealed system state can falls in regions:

1) $\mathrm{OO}, \mathrm{UO}, \mathrm{OU}$ and $\mathrm{UU}$, no maintenance is performed, only the system inspection cost $C_{\text {ins }}$ need to be paid and no maintenance time is consumed. The probability of this occurrence is :

$$
P_{M^{2}(0)}^{2}\left(x_{1}, x_{2}\right)=\int_{0}^{D_{p}^{(2)}} \int_{0}^{D_{p}^{(1)}} \pi_{2}\left(x_{1}, x_{2}\right) \mathrm{d} x_{1} \mathrm{~d} x_{2}
$$

2) $\mathrm{PU}$ or $\mathrm{UP}$, only unit 1 or unit 2 needs a preventive maintenance with probability $P_{\mathrm{PU}}^{2}\left(x_{1}, x_{2}\right)$ or $P_{\mathrm{UP}}^{2}\left(x_{1}, x_{2}\right)$. If the maintainable unit is $i(i=1,2)$, the unavailable duration for maintenance is $t_{p}^{(i)}$ and the maintenance costs is $C_{\text {ins }}+C_{s}+C_{p}^{(i)}+C_{d} \cdot t_{p}^{(i)}$.

3) $\mathrm{PO}, \mathrm{OP}$ and $\mathrm{PP}$, due to the assumption that the opportunistic maintenance of each unit incurs same cost and time as its preventive maintenance, in these scenarios, the unavailable duration for maintenance is $\max \left(t_{p}^{(1)}, t_{p}^{(2)}\right)$ and the maintenance costs can be expressed as $C_{i n s}+C_{s}+C_{p}^{(1)}+C_{p}^{(2)}+C_{d} \cdot \max \left(t_{p}^{(1)}, t_{p}^{(2)}\right)$. The probability is $P_{\mathrm{OP}}^{2}\left(x_{1}, x_{2}\right)+P_{\mathrm{PO}}^{2}\left(x_{1}, x_{2}\right)+P_{\mathrm{PP}}^{2}\left(x_{1}, x_{2}\right)$.

4) $\mathrm{CU}$ or UC, only unit 1 or unit 2 needs a corrective maintenance with probability $P_{\mathrm{CU}}^{2}\left(x_{1}, x_{2}\right)$ or $P_{\mathrm{UC}}^{2}\left(x_{1}, x_{2}\right)$. If the maintainable unit is $i(i=1,2)$, the unavailable duration for maintenance is $t_{c}^{(i)}$ and the maintenance costs is $C_{\text {ins }}+C_{s}+C_{c}^{(i)}+C_{d} \cdot t_{c}^{(i)}$.

5) OC and PC, the unavailable duration for maintenance is $\max \left(t_{p}^{(1)}, t_{c}^{(2)}\right)$ and its maintenance costs can be expressed as $C_{i n s}+C_{s}+C_{p}^{(1)}+C_{c}^{(2)}+C_{d} \cdot \max \left(t_{p}^{(1)}, t_{c}^{(2)}\right)$. The probability of its occurrence is $P_{\mathrm{OC}}^{2}\left(x_{1}, x_{2}\right)+P_{\mathrm{PC}}^{2}\left(x_{1}, x_{2}\right)$.

6) $\mathrm{CO}$ and $\mathrm{CP}$, the unavailable duration for maintenance is $\max \left(t_{c}^{(1)}, t_{p}^{(2)}\right)$ and its maintenance costs can be expressed as $C_{i n s}+C_{s}+C_{c}^{(1)}+C_{p}^{(2)}+C_{d} \cdot \max \left(t_{c}^{(1)}, t_{p}^{(2)}\right)$. The probability of its occurrence is $P_{\mathrm{CO}}^{2}\left(x_{1}, x_{2}\right)+P_{\mathrm{CP}}^{2}\left(x_{1}, x_{2}\right)$.

7) CC, the unavailable duration for maintenance is $\max \left(t_{c}^{(1)}, t_{c}^{(2)}\right)$ and the maintenance costs is $C_{\text {ins }}+C_{s}+C_{c}^{(1)}+C_{c}^{(2)}+C_{d} \cdot \max \left(t_{c}^{(1)}, t_{c}^{(2)}\right)$. The probability of its occurrence is $P_{\mathrm{CC}}^{2}\left(x_{1}, x_{2}\right)$.

If system failure occurs between two inspections, it contains two sub-scenarios:

1) If the system failure is triggered by failure of one unit $i(i=1$ or 2$)$. Its failure probability is $P\left(S_{i}^{\prime}=\tau_{f}^{k}\right)-P\left(S_{1}^{\prime}=\tau_{f}^{k}, S_{2}^{\prime}=\tau_{f}^{k}\right)$. A corrective maintenance is performed on unit $i$ and an inspection is performed on unit $j(j \neq i)$.

a) If $x_{j}<D_{o}^{(j)}$, the unit $j$ will not be maintained, the unavailable duration only contains corrective maintaining time for unit $i, t_{c}^{(i)}$, and the maintenance costs can be expressed as $C_{i n s}^{(j)}+C_{s}+C_{c}^{(i)}+C_{d} \cdot t_{c}^{(i)}$. The probability of its occurrence is:

$$
\int_{0}^{D_{0}^{(j)}} \int_{D_{f}^{(i)}}^{\infty} \pi_{2}\left(x_{1}, x_{2}\right) \mathrm{d} x_{i} \mathrm{~d} x_{j}
$$


b) If $D_{o}^{(j)} \leq x_{j}<D_{f}^{(j)}$, the unit $j$ will be preventively maintained simultaneously with unit $i$. Compared to the previous sub-scenario, the unavailable duration is the maximum of the corrective maintaining time for unit $i$, $t_{c}^{(i)}$, and the preventive maintenance time for unit $j, t_{p}^{(j)}$, then the maintenance costs can be expressed as $C_{\text {ins }}^{(j)}+C_{s}+C_{c}^{(i)}+C_{p}^{(j)}+C_{d} \cdot \max \left(t_{c}^{(i)}, t_{p}^{(j)}\right)$. The probability of its occurrence is:

$$
\int_{D_{o}^{(j)}}^{D_{f}^{(j)}} \int_{D_{f}^{(i)}}^{\infty} \pi_{2}\left(x_{1}, x_{2}\right) \mathrm{d} x_{i} \mathrm{~d} x_{j}
$$

2) If the system failure is triggered by failure of two units, its probability is $P\left(\mathrm{~S}_{1}{ }^{\prime}=\tau_{f}^{k}, \mathrm{~S}_{2}{ }^{\prime}=\tau_{f}^{k}\right)$. At that time, the entire system will be correctively maintained simultaneously. The unavailable duration for maintenance is $\max \left(t_{c}^{(1)}, t_{c}^{(2)}\right)$ and the maintenance costs is $C_{s}+C_{c}^{(i)}+C_{c}^{(j)}+C_{d} \cdot \max \left(t_{c}^{(1)}, t_{c}^{(2)}\right)$.

The expected total cost of a semi-regenerative cycle is concluded by Equation (38),

$$
\begin{aligned}
& E(C(S))=\left[1-\sum_{\tau_{f}^{k}=1}^{\tau_{k}-1} P\left(S=\tau_{f}^{k}\right)\right] \cdot\left\{C_{i n s} \cdot P_{M^{2}(0)}^{2}\left(x_{1}, x_{2}\right)+\left(C_{i n s}+C_{s}+C_{p}^{(1)}+C_{d} \cdot t_{p}^{(1)}\right) \cdot P_{\mathrm{PU}}^{2}\left(x_{1}, x_{2}\right)\right. \\
&+\left(C_{i n s}+C_{s}+C_{p}^{(2)}+C_{d} \cdot t_{p}^{(2)}\right) \cdot P_{\mathrm{UP}}^{2}\left(x_{1}, x_{2}\right)+\left[C_{i n s}+C_{s}+C_{p}^{(1)}+C_{p}^{(2)}+C_{d} \cdot \max \left(t_{p}^{(1)}, t_{p}^{(2)}\right)\right] \\
& \cdot\left[P_{\mathrm{PO}}\left(x_{1}, x_{2}\right)+P_{\mathrm{OP}}\left(x_{1}, x_{2}\right)+P_{\mathrm{PP}}\left(x_{1}, x_{2}\right)\right]+\left(C_{i n s}+C_{s}+C_{c}^{(1)}+C_{d} \cdot t_{c}^{(1)}\right) \cdot P_{\mathrm{CU}}^{2}\left(x_{1}, x_{2}\right) \\
&+\left(C_{i n s}+C_{s}+C_{c}^{(2)}+C_{d} \cdot t_{c}^{(2)}\right) \cdot P_{\mathrm{UC}}^{2}\left(x_{1}, x_{2}\right)+\left[C_{i n s}+C_{s}+C_{p}^{(1)}+C_{c}^{(2)}+C_{d} \cdot \max \left(t_{p}^{(1)}, t_{c}^{(2)}\right)\right] \cdot P_{\mathrm{PC}}^{2}\left(x_{1}, x_{2}\right) \\
&+\left[C_{i n s}+C_{s}+C_{c}^{(1)}+C_{p}^{(2)}+C_{d} \cdot \max \left(t_{c}^{(1)}, t_{p}^{(2)}\right)\right] \cdot P_{\mathrm{CP}}^{2}\left(x_{1}, x_{2}\right) \\
&\left.+\left[C_{i n s}+C_{s}+C_{c}^{(1)}+C_{c}^{(2)}+C_{d} \cdot \max \left(t_{c}^{(1)}, t_{c}^{(2)}\right)\right] \cdot P_{\mathrm{CC}}^{2}\left(x_{1}, x_{2}\right)\right\} \\
&+\sum_{\tau_{f}^{k}=1}^{\tau_{k}-1}\left\{\sum _ { i \neq j } [ P ( S _ { i } = \tau _ { f } ^ { k } ) - P ( S _ { 1 } = \tau _ { f } ^ { k } , S _ { 2 } = \tau _ { f } ^ { k } ) ] \cdot \left[\left(C_{i n s}^{(j)}+C_{s}+C_{c}^{(i)}+C_{d} \cdot t_{c}^{(i)}\right) \cdot \int_{0}^{D_{0}^{(j)}} \int_{D_{f}^{(i)}}^{\infty} \pi\left(x_{1}, x_{2}\right) \mathrm{d} x_{i} \mathrm{~d} x_{j}\right.\right. \\
&\left.+\left(C_{i n s}^{(j)}+C_{s}+C_{c}^{(i)}+C_{p}^{(j)}+C_{d} \cdot \max \left(t_{c}^{(i)}, t_{p}^{(j)}\right)\right) \cdot \int_{D_{o}^{(j)}}^{D_{f}^{(j)}} \int_{D_{f}^{(i)}}^{\infty} \pi\left(x_{1}, x_{2}\right) \mathrm{d} x_{i} \mathrm{~d} x_{j}\right] \\
&\left.+P\left(S_{1}=\tau_{f}^{k}, S_{2}=\tau_{f}^{k}\right) \cdot\left(C_{s}+C_{c}^{(1)}+C_{c}^{(2)}+C_{d} \cdot \max \left(t_{c}^{(1)}, t_{c}^{(2)}\right)\right)\right\}
\end{aligned}
$$

Based on the above analysis, the expected unavailable duration for maintenance over a semi-regenerative cycle can be expressed as Equation (39).

$$
\begin{aligned}
E(D)= & {\left[1-\sum_{\tau_{f}^{k}=1}^{\tau_{k}-1} P\left(S=\tau_{f}^{k}\right)\right] \cdot\left\{t_{p}^{(1)} \cdot P_{\mathrm{PU}}^{2}\left(x_{1}, x_{2}\right)+t_{p}^{(2)} \cdot P_{\mathrm{UP}}^{2}\left(x_{1}, x_{2}\right)+\max \left(t_{p}^{(1)}, t_{p}^{(2)}\right)\right.} \\
& \cdot\left[P_{\mathrm{PO}}\left(x_{1}, x_{2}\right)+P_{\mathrm{OP}}\left(x_{1}, x_{2}\right)+P_{\mathrm{PP}}\left(x_{1}, x_{2}\right)\right]+t_{c}^{(1)} \cdot P_{\mathrm{CU}}^{2}\left(x_{1}, x_{2}\right)+t_{c}^{(2)} \cdot P_{\mathrm{UC}}^{2}\left(x_{1}, x_{2}\right) \\
& \left.+\max \left(t_{p}^{(1)}, t_{c}^{(2)}\right) \cdot P_{\mathrm{PC}}^{2}\left(x_{1}, x_{2}\right)+\max \left(t_{c}^{(1)}, t_{p}^{(2)}\right) \cdot P_{\mathrm{CP}}^{2}\left(x_{1}, x_{2}\right)+\max \left(t_{c}^{(1)}, t_{c}^{(2)}\right) \cdot P_{\mathrm{CC}}^{2}\left(x_{1}, x_{2}\right)\right\} \\
& +\sum_{\tau_{f}^{k}=1}^{\tau_{k}-1}\left\{\sum _ { i \neq j } [ P ( S _ { i } = \tau _ { f } ^ { k } ) - P ( S _ { 1 } = \tau _ { f } ^ { k } , S _ { 2 } = \tau _ { f } ^ { k } ) ] \cdot \left[t_{c}^{(i)} \cdot \int_{0}^{D_{o}^{(j)}} \int_{D_{f}^{(i)}}^{\infty} \pi\left(x_{1}, x_{2}\right) \mathrm{d} x_{i} \mathrm{~d} x_{j}\right.\right. \\
& \left.\left.+\max \left(t_{c}^{(i)}, t_{p}^{(j)}\right) \cdot \int_{D_{0}^{(j)}}^{D_{f}^{(j)}} \int_{D_{f}^{(i)}}^{\infty} \pi\left(x_{1}, x_{2}\right) \mathrm{d} x_{i} \mathrm{~d} x_{j}\right]+P\left(S_{1}=\tau_{f}^{k}, S_{2}=\tau_{f}^{k}\right) \cdot \max \left(t_{c}^{(1)}, t_{c}^{(2)}\right)\right\}
\end{aligned}
$$

The established model, as shown in (27) is a non-linear, single-objective optimization model with a mixture 
of constraint variables. A genetic algorithm (GA) is chosen as an optimization method to solve the model because it can deal with linear and nonlinear, constrained and non-constrained, as well as discrete, continuous, and hybrid search spaces.

\section{Numerical Experiments}

\subsection{Experimental Results}

A numerical example is presented herein to demonstrate the correctness and validation of the DSSP method. A gamma distribution is often used to characterize continuous wear processes with non-negative, stationary, and statistically independent increments starting from zero level [9] [17]. An advantage of the gamma distribution process is the existence of an explicit probability distribution function which permits feasible mathematical developments [18]. Therefore, we assume that the deterioration increments of unit $i, \Delta x_{k}^{(i)}$, follow the gamma distribution, $\Gamma\left(\alpha_{i}, \beta_{i}\right)$. Its probability density function can be expressed as:

$$
\begin{aligned}
& f_{i}\left(x \mid \alpha_{i}, \beta_{i}\right)=\frac{\beta_{i}^{\alpha_{i}}}{\Gamma\left(\alpha_{i}\right)} x^{\alpha_{i}-1} \mathrm{e}^{-\beta_{i} x}, x \geq 0 \\
& \Gamma\left(\alpha_{i}\right)=\int_{0}^{\infty} x^{\alpha_{i}-1} \mathrm{e}^{-x} \mathrm{~d} x, \alpha_{i}>0
\end{aligned}
$$

A gamma wear process in continuous time $z$ follows the gamma distribution $\Gamma\left(z \alpha_{i}, \beta_{i}\right)$.

The $k$ th inspection interval $\tau_{k}$ is defined as a function of the system deterioration state after the previous maintenance decision, $m\left(x_{1}^{(k-1)}, x_{2}^{(k-1)}\right)$, where $m(\cdot)$ is a decreasing function from 0 to $T$ (Grall, Dieulle et al. 2002). In the numerical experiment, we define $m\left(x_{1}, x_{2}\right)=\left(1-a_{1} x_{1}-a_{2} x_{2}\right) T$ as an experimental function to ensure the maximum inspection interval is $T$. Therefore, determination of the optimal $\tau_{k}$ is to determine the optimal value of the coefficients $a_{1}$ and $a_{2}$.

For two non-identical units, we suppose that: $\alpha_{1}=1, \beta_{1}=1.5, \alpha_{2}=2, \beta_{2}=2, D_{f}^{(1)}=4, D_{f}^{(2)}=5$. When $\tau_{k}=3, D_{o}^{(1)}=2.5, D_{o}^{(2)}=3.5, D_{p}^{(1)}=3.5, D_{p}^{(2)}=4$, and truscated data is defined as $6 D_{f}^{(1)}$ and $6 D_{f}^{(2)}$ instead of $\infty$, approximate numerical solution of $\pi\left(x_{1}, x_{2}\right)$ is shown in Figure 6.

We will verify the proposed deterioration state space partition method by making a decision table and performing sensitivity analysis of different parameters. Additionally suppose that $C_{p}^{(1)}=40, C_{p}^{(2)}=50, C_{c}^{(1)}=100$,

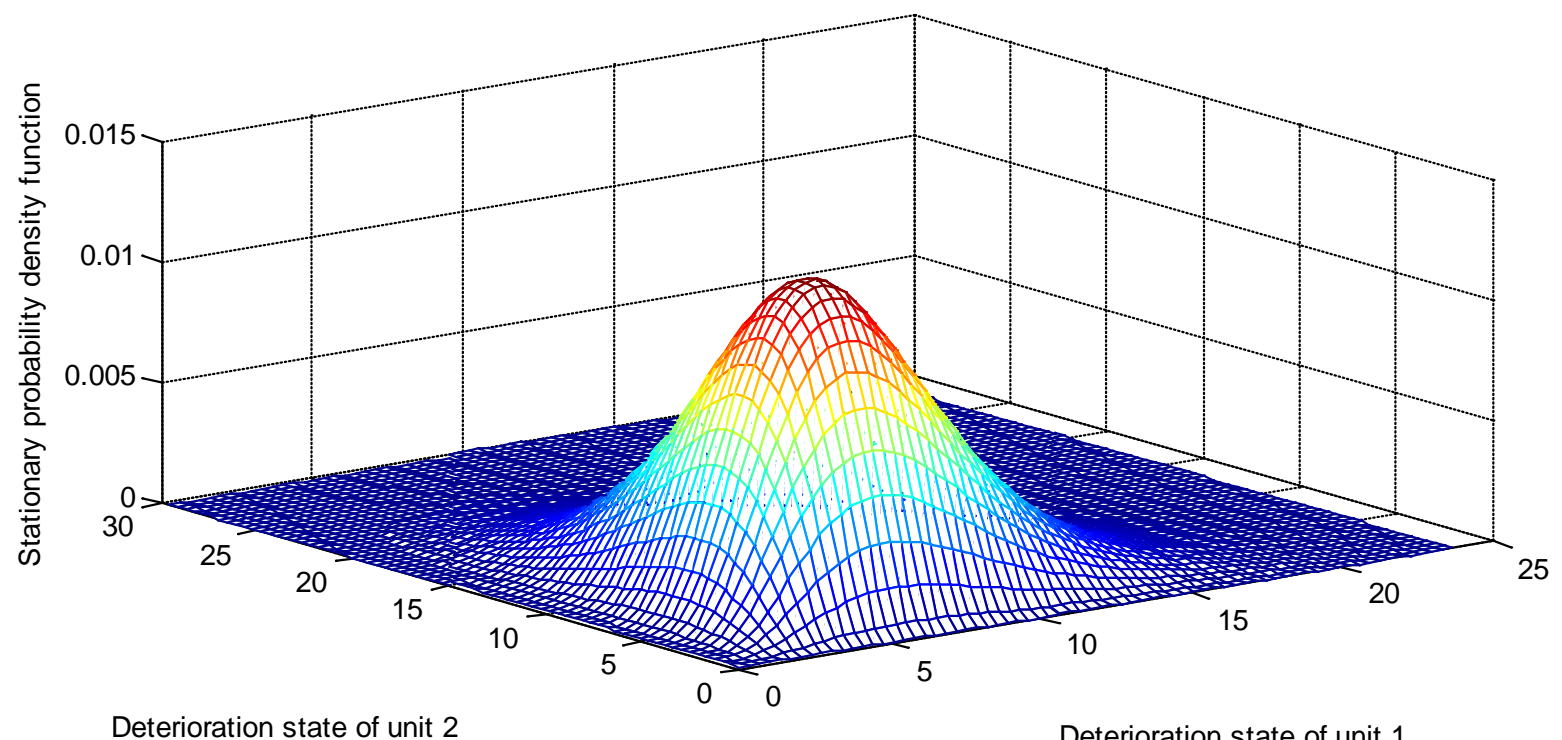

Figure 6. Example of deterioration and maintenance evolution of a two-unit system. 
$C_{c}^{(2)}=300, t_{p}^{(1)}=0.5, t_{p}^{(2)}=1, t_{c}^{(1)}=2, t_{c}^{(2)}=4, C_{s}=50, C_{d}=100, C_{\text {ins }}^{(1)}=2, C_{\text {ins }}^{(2)}=3$, then $C_{\text {ins }}=5$, and $T=10$. The approximate optimal value of the expected costs per unit time is obtained using GA with parameters set as follows: population size is 20 , maximum number of generations is 50 , generation gap is 0.8 , crossover rate is 0.8 , and mutation rate is 0.2 . Figure 7 shows an example of the evolution of the optimization process. The optimal values of the decision variables are $\alpha_{1}=0.295521, \alpha_{1}=0.297887, D_{p}^{(1)}=2.683018$, $D_{p}^{(2)}=3.461388, D_{o}^{(1)}=1.003265, D_{o}^{(2)}=2.920907$ corresponding to the minimal cost rates $C R(t)=85.671100$.

\subsection{Sensitivity Analysis}

From the definition of $\pi_{2}\left(x_{1}, x_{2}\right)$, we discover that adjustment of the length of the inspection cycle and opportunistic and preventive maintenance thresholds can result in different stationary probability density functions. Further, it can influence the probabilities of all possible maintenance groups. In a specific maintenance optimization model, it can obtain different specific target values for different choices. For facilitation, we analyze the effects of the inspection interval on the expected duration of a semi-regenerative cycle and the expected cost per unit time with a deterministic value $T$. Their relations are presented in Figure 8. We can observe from Figure 8(a) that $E(S)$ increases with increasing inspection period $T$. However, its rate of growth reduced gradually and reached an asymptote at a certain time. Because the probability that random failure occurs in an inspection interval is lower when $T$ is smaller, the expected duration of a semi-regenerative cycle approaches the length of the inspection period. When $T$ becomes larger, the probability that a random failure occurs in an inspection interval increases. Then, the expected duration of a semi-regenerative cycle gradually closes to the natural failure period of the system. The results in Figure 8(b) indicate minimal values of expected costs per unit time with different values of $T$. It can be observed from the this figure that expected costs rate increase with increasing $T$ after their optimal values, due to the increase in the corrective maintenance costs and the probability that random failure occurs in an inspection interval. However, the growth curve of the expected cost rate becomes smooth when $T$ becomes too large because the expected duration of a semi-regenerative cycle gradually closes to the natural failure period of the system.

Next, we focus on the influence of the opportunistic and preventive maintenance thresholds of each unit over the optimal values. When $T=5, D_{o}^{(i)}=D_{p}^{(i)}(i=1,2)$, and $D_{p}^{(i)}$ varies from 0 to $D_{f}^{(i)}$, the results in Figure 9(a) show minimal values of expected total costs with different preventive maintenance thresholds. When $T=5, D_{p}^{(1)}=3.5, D_{p}^{(2)}=4.5$, and $D_{o}^{(i)}$ varies from 0 to $D_{p}^{(i)}$, the results in Figure $9(\mathrm{~b})$ show minimal

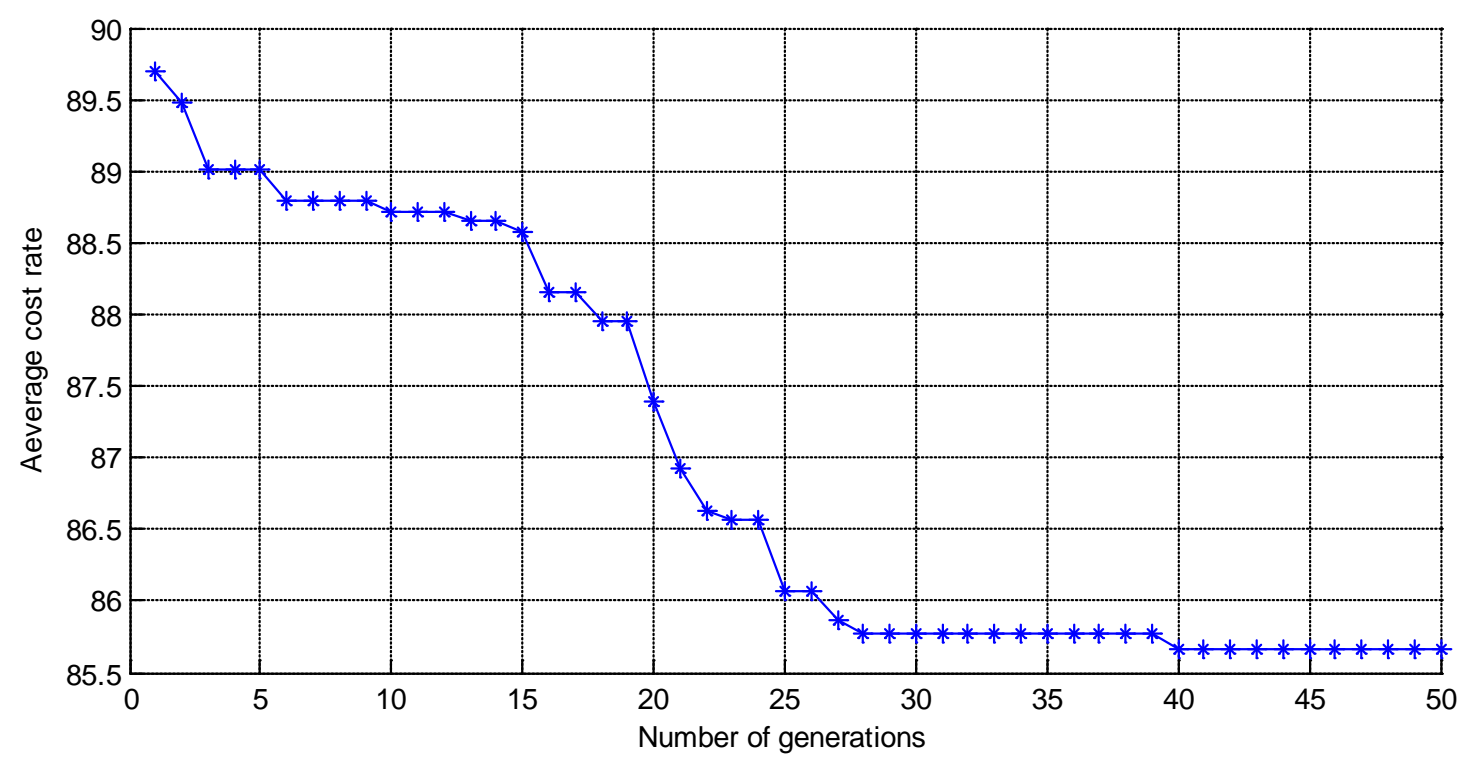

Figure 7. An example of optimization result of GA. 


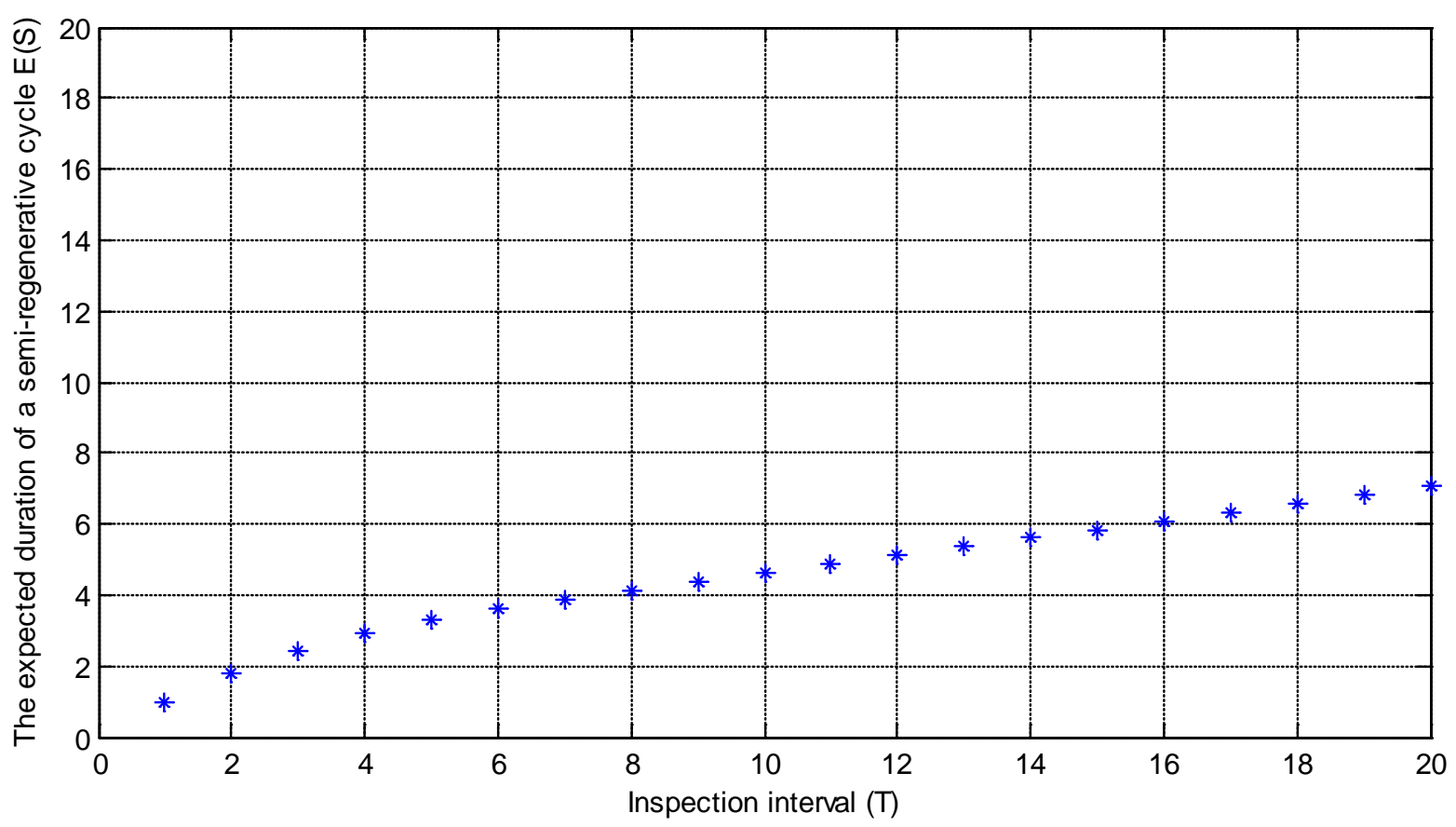

(a)

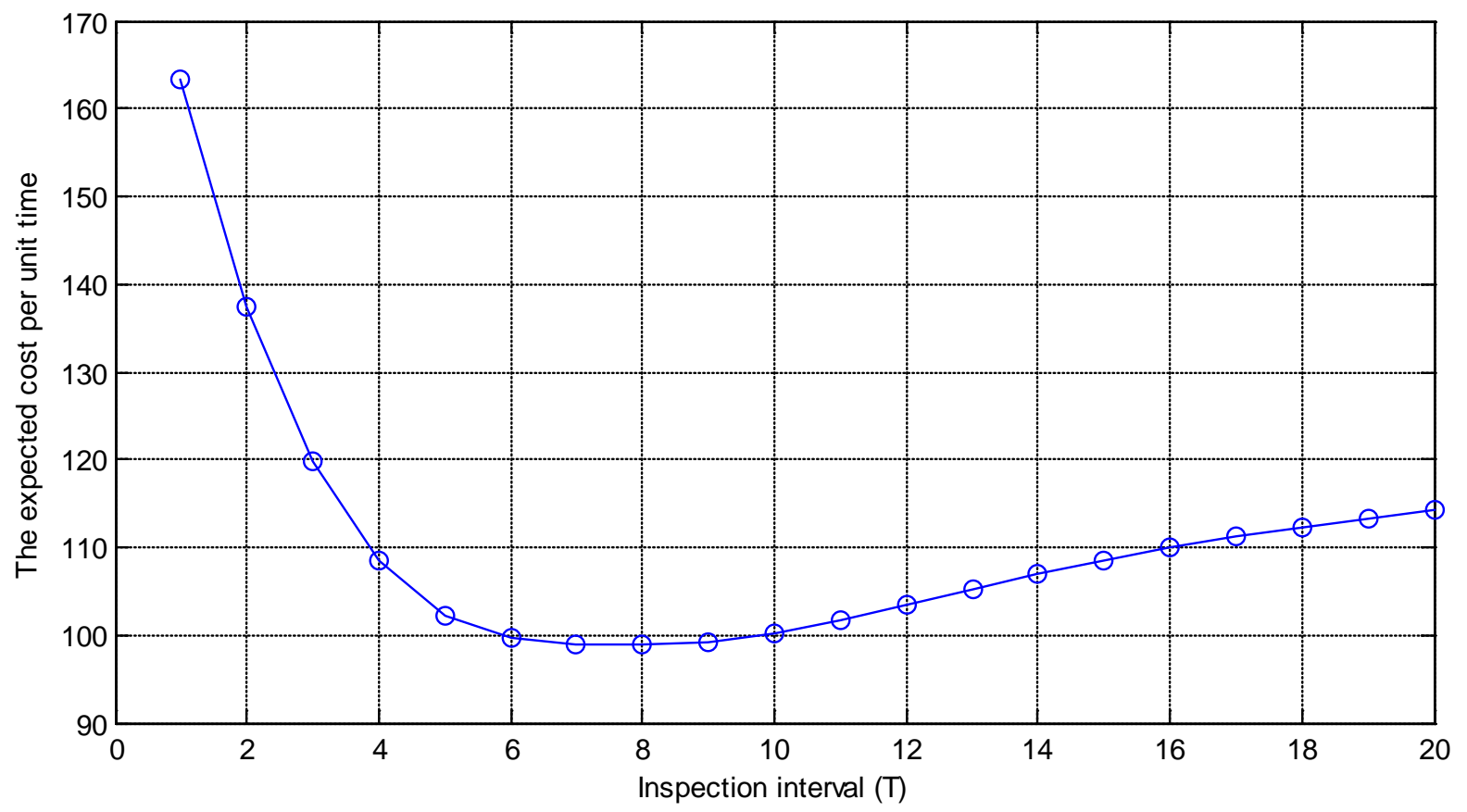

(b)

Figure 8. Effects of inspection interval on the optimization objective. (a) On the expected duration of a semi-regenerative cycle; (b) On the expected cost per unit time.

values of expected total costs with different opportunistic maintenance thresholds. Figure 9 indicate that the cost rate is relatively small at smaller $D_{p}^{(i)}$ and $D_{o}^{(i)}$ due to the higher probability of preventive maintenance and lower probability of random failure, and combined with the fact that preventive maintenance costs are less than corrective maintenance costs. But if $D_{p}^{(i)}$ and $D_{o}^{(i)}$ is too small, frequent preventive maintenance also incurs high costs. With the increase in preventive maintenance thresholds, preventive maintenance zone gradu- 
ally becomes narrow, and then the probability of preventive maintenance is reduced. Thus the probability of failure within an inspection period rises and the average cost rate increases significantly due to the influence of higher corrective maintenance costs.

On account of two key issues of this paper, the opportunistic preventive maintenance structure and the nonnegligible maintenance times, we further analyze the influence of the cost parameters $C_{s}$ and $C_{d}$ over the optimal maintenance policy. Table 1 and Table 2 show the results of sensitivity experiments. In these tables, Zone $e_{o}^{(1)}$, Zone $e_{o}^{(2)}$, Zone $e_{p}^{(1)}$ and Zone $e_{p}^{(2)}$ denote the widths of the opportunistic and preventive maintenance zones for unit 1 and 2.

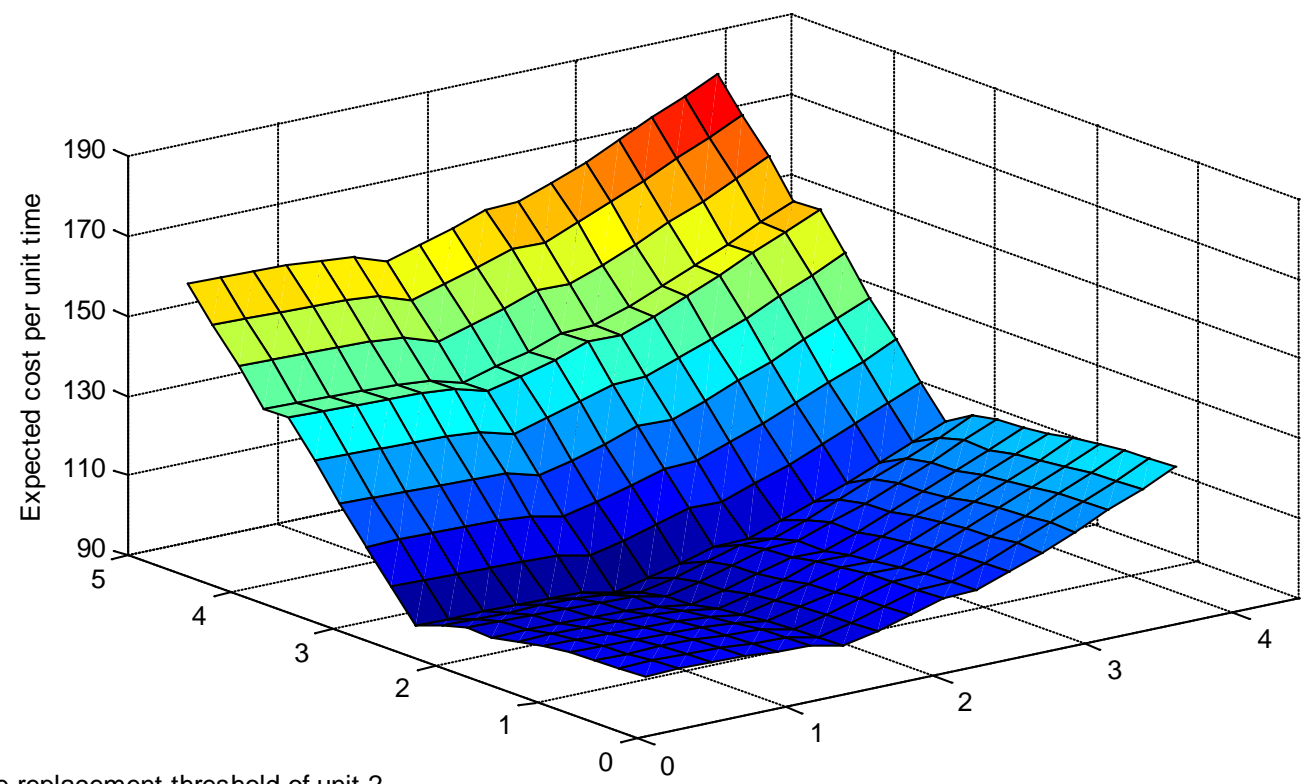

Preventive replacement threshold of unit 2

Preventive replacement threshold of unit 1

(a)

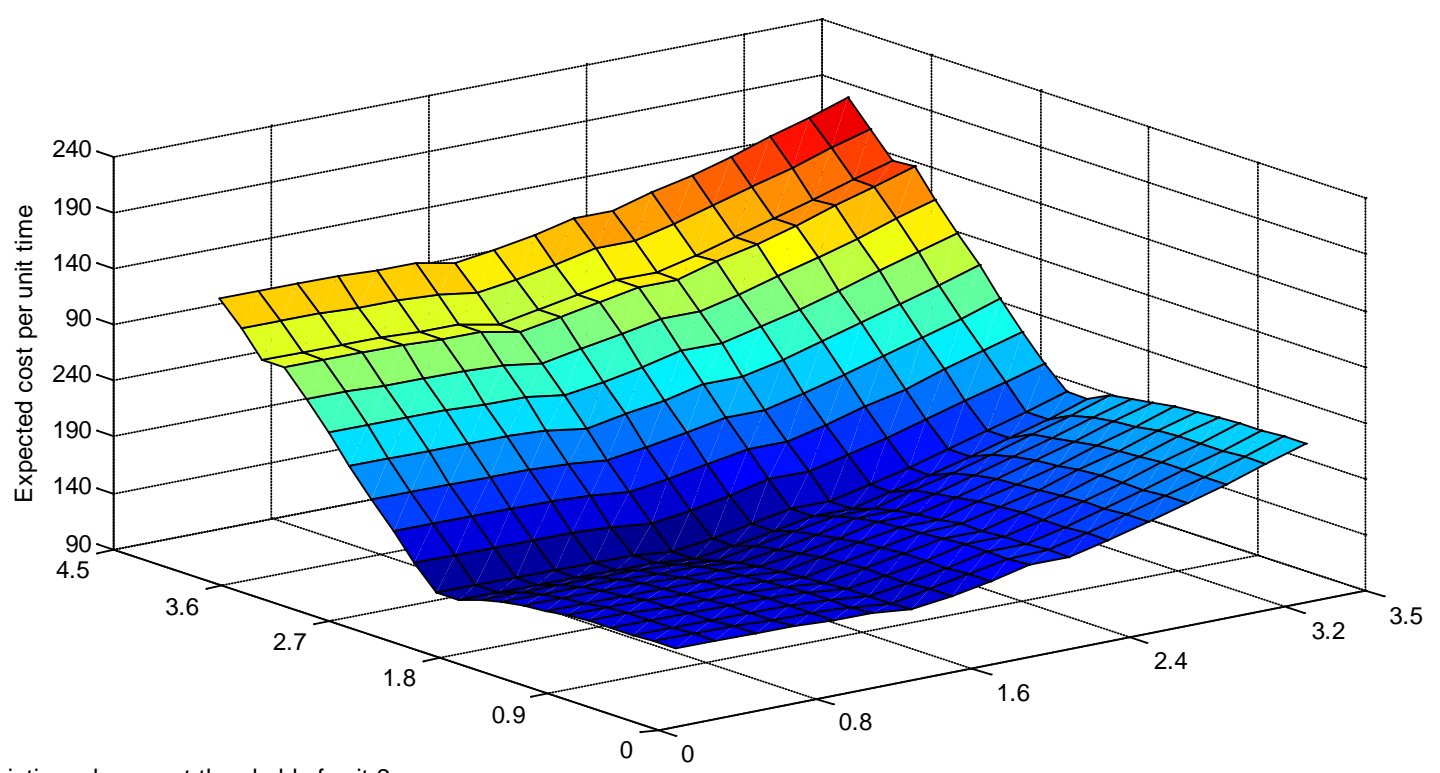

Opportunistic replacement threshold of unit 2

Opportunistic replacement threshold of unit 1

(b)

Figure 9. Influence of maintenance thresholds over the expected cost per unit time. (a) Preventive maintenance thresholds; (b) Opportunistic maintenance thresholds. 
Table 1. Influence of $C_{s}$ over the optimal strategy, where $C_{p}^{(1)}=40, C_{p}^{(2)}=50, C_{f}^{(1)}=100, C_{f}^{(2)}=300, C_{d}=100$.

\begin{tabular}{cccccccccccc}
\hline$C_{\mathrm{s}}$ & $\mathrm{CR}(t)$ & $a_{1}$ & $a_{2}$ & $D_{p}^{(1)}$ & $D_{p}^{(2)}$ & $D_{o}^{(1)}$ & $D_{o}^{(2)}$ & Zone $_{o}^{(1)}$ & ${\text { Zone } e^{(2)}}_{\text {Zone }}^{(1)}$ & Zone $_{p}^{(2)}$ \\
\hline 5 & 77.088050 & 0.351357 & 0.498958 & 3.237510 & 3.546651 & 2.763565 & 2.013934 & 0.473945 & 1.532717 & 0.76249 & 1.453349 \\
50 & 85.671100 & 0.295521 & 0.297887 & 3.949277 & 3.728501 & 2.687689 & 2.383558 & 1.261588 & 1.344943 & 0.050723 & 1.271499 \\
100 & 162.998650 & 0.100000 & 0.194674 & 3.834360 & 3.646004 & 2.139388 & 2.336465 & 1.694972 & 1.309539 & 0.16564 & 1.353996 \\
\hline
\end{tabular}

Table 2. Influence of $C_{d}$ over the optimal strategy, where $C_{s}=50, C_{p}^{(1)}=40, C_{p}^{(2)}=50, C_{f}^{(1)}=100, C_{f}^{(2)}=300$, $C_{d}=100$.

\begin{tabular}{|c|c|c|c|c|c|c|c|c|c|c|c|}
\hline$C_{d}$ & $C R(t)$ & $a_{1}$ & $a_{2}$ & $D_{p}^{(1)}$ & $D_{p}^{(2)}$ & $D_{o}^{(1)}$ & $D_{o}^{(2)}$ & Zone $_{o}^{(1)}$ & Zone $_{o}^{(2)}$ & Zone $_{p}^{(1)}$ & Zone $_{p}^{(2)}$ \\
\hline 10 & 46.292150 & 0.306901 & 0.319461 & 2.851902 & 4.375703 & 2.686558 & 2.708539 & 0.165344 & 1.667164 & 1.148098 & 0.624297 \\
\hline 100 & 85.671100 & 0.295521 & 0.297887 & 3.949277 & 3.728501 & 2.687689 & 2.383558 & 1.261588 & 1.344943 & 0.050723 & 1.271499 \\
\hline 500 & 259.876200 & 0.174899 & 0.250782 & 3.437280 & 3.682932 & 2.666667 & 2.154653 & 0.770613 & 1.528279 & 0.56272 & 1.317068 \\
\hline
\end{tabular}

Results from Table 1 indicate that with increasing of set-up costs, the cost of all maintenance activities in crease. In order to reduce expected costs rate, the frequency of maintenance must be reduced. The corrective maintenance cannot get an optimal decision, therefore this objective only can be achieved by reduce the frequency of preventive maintenance. The result is the width of preventive maintenance zones of all units are decreased with the increase of set-up costs. However, it is not wise to increase the preventive maintenance threshold blindly when set-up costs are very large. It will incur a large probability and cost of corrective maintenance. So the inspection interval becomes small with continuous increasing of set-up costs. With increasing of set-up costs, significance of opportunistic maintenance is more obvious, thus the width of preventive maintenance zones of all units are increased.

As shown in Table 2, with increasing costs of the downtime losses, $C_{d}$, the opportunistic and preventive maintenance thresholds of all units decrease to reduction in the probabilities and costs of corrective maintenance and thus resulting in an overall reduction in the cost rate. There are no evident trends in the change of widths of the opportunistic maintenance zones of units 1 and 2 with change in $C_{d}$. This is because the relationship between $C_{d}$ and maintenance zones is not direct.

\section{Conclusions}

In order to satisfy the objective-specific optimization modeling requirement, the DSSP method, which is presented in our recent study to analyze possible maintenance groups at each maintenance decision point and deduce their corresponding probabilities, is extended by detailing its partition with maintenance type due to the differences in costs and time consumptions of different maintenance activities. A more detailed partition method is presented and a new representation of the possible maintenance requirements and their corresponding probabilities are derived according to the partition results. In addition, focusing on two-unit systems with random "hard failure" and non-negligible maintenance time, a non-periodical condition-based opportunistic preventive-maintenance structure was presented. Utilizing the proposed method, a long-term average cost model is established based on semi-renewal theory. The optimal inspection period function of the system, the opportunities and the preventive maintenance thresholds of each unit are determined jointly. Numerical experiments are conducted to evaluate the proposed method. The results show that: An optimal maintenance strategy can be obtained by solving the optimization cost model, which is established using the proposed DSSP method. Together with the results of sensitivity analysis of the cost parameters, it indicates that the proposed modeling method is correct and well performed to optimize maintenance policies for two-unit systems.

According to the proposed method, the solution of the stationary law of system state $\pi\left(x_{1}, \cdots, x_{n}\right)$ is the key 
issue for evaluation of such criterion requires determination. However, the expression of the stationary probability density for multi-unit system with $n$ units can be reorganized as a set of $i_{\max }^{n}$ linear algebraic equations in $i_{\max }^{n} \times i_{\max }^{n}$ unknowns in form of $\boldsymbol{A x}=\boldsymbol{b}$, where the dimension of $\boldsymbol{A}$ is $i_{\max }^{n} \times i_{\max }^{n}$. It indicates that the dimension of the solution matrix grows exponentially when the number of units in a multi-unit system increases. Even for a multi-unit system with a few units, if the number of portions for numerical integration $i_{\max }$ increases continuously, the problem of "out of memory" may occur. In the future, this problem can be solved in two ways. First, the numerical solution of the stationary law of system state $\pi\left(x_{1}, \cdots, x_{n}\right)$ warrants further study by introducing new technologies and methods, such as code optimization, sparse matrix storage and improved method for solving linear equations by analyzing the special characteristics of its matrix of coefficients. Second, it can be calculated with the aid of high performance computer and parallel computer.

In addition, further studies are needed considering more realistic systems and difficult maintenance models. These should include maintenance models with imperfect maintenance, models taking into account punishment for untimely preventive maintenance, and models for evaluation of additional performance criteria of the proposed policy (e.g., system reliability and availability), etc.

\section{Acknowledgements}

This project is supported by the National Natural Science Foundation of China (General Program) (Grant No. 61573250) and the PhDResearch Startup Foundation of Taiyuan University of Science \& Technology (Grant No. 20152021).

\section{References}

[1] Moghaddam, K.S. and Usher, J.S. (2011) Preventive Maintenance and Replacement Scheduling for Repairable and Maintainable Systems Using Dynamic Programming. Computers \& Industrial Engineering, 60, 654-665. http://dx.doi.org/10.1016/j.cie.2010.12.021

[2] Nowakowski, T. and Werbińka, S. (2009) On Problems of Multi-Component System Maintenance Modelling. International Journal of Automation and Computing, 6, 364-378. http://dx.doi.org/10.1007/s11633-009-0364-4

[3] Cho, D.I. and Parlar, M. (1991) A Survey of Maintenance Models for Multi-Unit Systems. European Journal of Operational Research, 51, 1-23. http://dx.doi.org/10.1016/0377-2217(91)90141-H

[4] Wang, H. (2002) A Survey of Maintenance Policies of Deteriorating Systems. European Journal of Operational Research, 139, 469-489. http://dx.doi.org/10.1016/S0377-2217(01)00197-7

[5] Dekker, R., Wildeman R. and Duyn Schouten, F. (1997) A Review of Multi-Component Maintenance Models with Economic Dependence. Mathematical Methods of Operations Research, 45, 411-435. http://dx.doi.org/10.1007/BF01194788

[6] Nicolai, R. and Dekker, R. (2008) Optimal Maintenance of Multi-Component Systems: A Review, in Complex System Maintenance Handbook. Springer, London. http://dx.doi.org/10.1007/978-1-84800-011-7_11

[7] Van der Duyn Schouten, F.A. and Vanneste, S.G. (1990) Analysis and Computation of (n, N)-Strategies for Maintenance of a Two-Component System. European Journal of Operational Research, 48, 260-274. http://dx.doi.org/10.1016/0377-2217(90)90379-P

[8] Zheng, X. and Fard, N. (1992) Hazard-Rate Tolerance Method for an Opportunistic-Replacement Policy. IEEE Transactions on Reliability, 41, 13-20. http://dx.doi.org/10.1109/24.126664

[9] Castanier, B., Grall, A. and Bérenguer, C. (2005) A Condition-Based Maintenance Policy with Non-Periodic Inspections for a Two-Unit Series System. Reliability Engineering \& System Safety, 87, 109-120. http://dx.doi.org/10.1016/j.ress.2004.04.013

[10] Nguyen, K.-A., Do, P. and Grall, A. (2014) Condition-Based Maintenance for Multi-Component Systems Using Importance Measure and Predictive Information. International Journal of Systems Science, 1, 228-245. http://dx.doi.org/10.1080/23302674.2014.983582

[11] Koochaki, J., Bokhorst, J.A., Wortmann, H. and Klingenberg, W. (2012) Condition Based Maintenance in the Context of Opportunistic Maintenance. International Journal of Production Research, 50, 6918-6929. http://dx.doi.org/10.1080/00207543.2011.636924

[12] Jinqiu, H. and Laibin, Z. (2013) Opportunistic Maintenance Strategy for Complex Oil and Gas Production Equipment Based on Fault Preventive Defense. Journal of mechanical engineering, 49, 167-175. (In Chinese)

[13] Zhang, Z., Wu, S., Li, B. and Lee, S. (2015) (n, N) Type Maintenance Policy for Multi-Component Systems with Fail- 
ure Interactions. International Journal of Systems Science, 46, 1051-1064. http://dx.doi.org/10.1080/00207721.2013.807386

[14] Zhang, X. and Zeng, J. (2014) Deterioration State Space Partitioning Method for Opportunistic Maintenance Modelling of Identical Multi-Unit Systems. International Journal of Production Research, 53, 2100-2118. http://dx.doi.org/10.1080/00207543.2014.965354

[15] Zhang, X. and Zeng, J. (2015) A General Modeling Method for Opportunistic Maintenance Modeling of Multi-Unit Systems. Reliability Engineering \& System Safety, 140, 176-190. http://dx.doi.org/10.1016/j.ress.2015.03.030

[16] Eto, H. and Dohi, T. (2006) Determining the Optimal Software Rejuvenation Schedule via Semi-Markov Decision Process. Journal of Computer Science, 2, 528. http://dx.doi.org/10.3844/jcssp.2006.528.534

[17] Wang, L., Chu J. and Mao, W. (2008) A Condition-Based Order-Replacement Policy for a Single-Unit System. Applied Mathematical Modelling, 32, 2274-2289. http://dx.doi.org/10.1016/j.apm.2007.07.016

[18] Zhu, Q., Peng, H. and van Houtum, G.-J. (2012) A Condition-Based Maintenance Policy for Multi-Component Systems with a High Maintenance Setup Cost. BETA Working Paper 400, Eindhoven University of Technology, Eindhoven. 


\section{Nomenclature}

$x_{t}^{(i)}$

$\mathbf{X}_{i}(t)$

$\left(\mathbf{X}_{k}^{(i)}\right)_{k \in \mathrm{N}}$

$\Delta x_{k}^{(i)}$

$f_{i}(x)$

$f_{i}^{(n)}(x)$

$D_{f}^{(i)}$

$D_{p}^{(i)}$

$D_{o}^{(i)}$

$\mathrm{U}$

$\mathrm{O}$

$\mathrm{P}$

C

$A_{1} A_{2} \cdots A_{n}$

$\pi_{n}\left(x_{1}, \cdots, x_{n}\right)$

$P_{A_{1} A_{2} \cdots A_{n}}^{n}$

$C_{\text {ins }}$

$C_{\text {ins }}^{(1)}$

$C_{\text {ins }}^{(2)}$

$C_{c}^{(i)}$

$C_{p}^{(i)}$

$t_{c}^{(i)}$

$t_{p}^{(i)}$

$C_{d}$

$\mathrm{C}_{\mathrm{s}}$

$T_{k}(k \in \mathrm{N})$

$R_{i}$

$S_{i}$

$S$

$S^{\prime}$

$D$

$C R(t)$

$C(S)$

$\tau_{k}$

$H_{i}\left(\tau_{f}^{k} \mid y_{i}^{\prime} ; \tau_{k}\right)$

$m\left(x_{1}^{(k-1)}, x_{2}^{(k-1)}\right)$

$E(\bullet)$ random variable represents deterioration state of unit $i$ at time $t$

deterioration process of unit $i$

deterioration process on an infinite discrete time grid $t_{k}(k \in \mathrm{N})$

increment of deterioration state of unit $i$ between $t_{k-1}$ and $t_{k}$

pdf of the increment of deterioration state $\Delta x_{k}^{(i)}$

pdf of the increments during $n$ units of time

threshold of hard failure of unit $i$

threshold of preventive maintenance of unit $i$

threshold of opportunistic maintenance of unit $i$

operating zone

opportunistic maintenance zone

preventive maintenance zone

corrective maintenance zone

maintenance activity list

joint probability density function of the system deterioration

probability of the maintenance activity $A_{1} A_{2} \cdots A_{n}$

inspection cost for the whole system

inspection cost for unit 1

inspection cost for unit 2

cost for a corrective maintenance of unit $i$

cost for a preventive maintenance of unit $i$

time incurred by a corrective maintenance of unit $i$

time incurred by a preventive maintenance of unit $i$

cost loss per unit time incurred by the system in in shutdown state set-up cost for each maintenance intervention

scheduled inspection points

regenerative point

semi-regenerative point

the length of a semi-regeneration cycle

uptime interval in a semi-regeneration cycle

downtime for maintenance in a semi-regeneration cycle

average cost of an infinite time $t$

total cost of the semi-regeneration cycle

the $k$ th inspection interval

probability that the $k$ th failure time of component $i$ is $\tau_{f}^{k}$ time units after the last semi-regeneration point given that the revealed deterioration state equal $y_{i}^{\prime}$ and the next inspection was scheduled $\tau_{k}$

function to calculate $k$ th inspection interval $\tau_{k}$

expectation value of • 


\section{Submit or recommend next manuscript to SCIRP and we will provide best service for you:}

Accepting pre-submission inquiries through Email, Facebook, Linkedin, Twitter, etc A wide selection of journals (inclusive of 9 subjects, more than 200 journals)

Providing a 24-hour high-quality service

User-friendly online submission system

Fair and swift peer-review system

Efficient typesetting and proofreading procedure

Display of the result of downloads and visits, as well as the number of cited articles

Maximum dissemination of your research work

Submit your manuscript at: http://papersubmission.scirp.org/ 